Boletín de la Sociedad Geológica Mexicana

Volumen Conmemorativo del Centenario

Grandes Fronteras Tectónicas de MéXico

Tomo LVII, NÚM. 1, 2005, P. 65-82

\title{
El sistema de fallas Taxco-San Miguel de Allende y la Faja Volcánica Transmexicana, dos fronteras tectónicas del centro de México activas durante el Cenozoico
}

\author{
Susana A. Alaniz-Álvarez*, y Ángel Francisco Nieto-Samaniego \\ Centro de Geociencias, Universidad Nacional Autónoma de México, \\ Campus Juriquilla, Apartado Postal 1-742, Querétaro, Qro., 76001, México. \\ *alaniz@geociencias.unam.mx
}

\section{Resumen}

La Faja Volcánica Transmexicana se ha reconocido como un arco volcánico que atraviesa México de la costa pacífica al Golfo de México, con actividad volcánica y fallamiento normal desde el Mioceno al Reciente. Se ha planteado que la oblicuidad del arco volcánico con la trinchera es debida a la geometría de la subducción, cuyo ángulo de inclinación varía haciéndose menor hacia el oriente. Por otra parte, se ha propuesto que la Faja Volcánica Transmexicana se ubica sobre una zona de debilidad preexistente. En este trabajo se discuten los eventos de deformación cenozoicos ocurridos al norte y sur de la Faja Volcánica Transmexicana para identificar el tiempo en el que ésta actuó como zona de discontinuidad y también para determinar la presencia del campo de deformación precursor del emplazamiento del arco volcánico. Utilizamos como una de las referencias el sistema de fallas Taxco-San Miguel de Allende, ya que es la única estructura reconocida que atraviesa el arco volcánico y de la cual se ha estudiado con detalle su evolución estructural. En la Mesa Central, al norte de la Faja Volcánica Transmexicana, la deformación extensional postlaramídica ha ocurrido en tres eventos relativamente discretos durante el Eoceno, Oligoceno y Mioceno-Reciente. Los tres eventos liberaron extensión en dos direcciones principales horizontales y acortamiento vertical. Del evento eocénico las direcciones principales de deformación son poco conocidas. El evento del Oligoceno fue el más importante y la mayor extensión fue de $20 \%$ hacia el ENE-WSW; en el tercer evento, activo desde el Mioceno, la deformación ha estado concentrada principalmente en la Faja Volcánica Transmexicana y en su límite norte con la Mesa Central. La deformación extensional oligocénica de la Mesa Central migró hacia el poniente hasta culminar con la apertura del Golfo de California y hacia el sur-oriente hacia la Faja Volcánica Transmexicana. En la Sierra Madre Oriental, al oriente de la Mesa Central y norte de la Faja Volcánica Transmexicana, la deformación cenozoica ha sido mínima. Al sur de la Faja Volcánica Transmexicana, en la región de Taxco, Gro., se han documentado dos eventos de deformación postlaramídicos acomodados principalmente por fallas laterales NW-SE y N-S. El primero ocurrió durante el Eoceno tardío con una dirección de extensión NNW-SSE y acortamiento hacia el ENE-WSW. El segundo evento ocurrió durante el Oligoceno temprano, la extensión máxima ocurrió hacia el NE-SW y el acortamiento hacia el NW-SE, ambos eventos migraron hacia el oriente.

La mayor parte de las rocas volcánicas a lo largo de la Faja Volcánica Transmexicana son rocas volcánicas miocénicas, sin embargo se ha reportado la presencia de rocas volcánicas oligocénicas en la mitad inferior del relleno de la cuenca de México; por otro lado este arco volcánico se encuentra alojado en cuencas tectónicas limitadas por fallas mayores. Para la cuenca de México se infiere que la actividad de las fallas que limitan a la Faja Volcánica Transmexicana inició en el Eoceno tardío. Se concluye que desde el Eoceno el estilo de la deformación de la Mesa Central y de la Sierra Madre del Sur ha sido distinto, esto implica la existencia de una zona de despegue entre estas provincias. A partir del Oligoceno la actividad de las fallas laterales en la Sierra Madre del Sur produjeron acortamiento en la dirección principal NW-SE, 
generando en la zona de despegue una deformación con extensión oblicua a la dirección del arco volcánico y una componente de cizalla izquierda. Los eventos de deformación anteriores al Mioceno formaron una zona de despegue en la cual se desarrolló la cuenca de México y además en esa misma zona se canalizaron hacia la superficie los magmas que formaron la parte central de la Faja Volcánica Transmexicana.

Palabras clave: Faja Volcánica Transmexicana, fallas mayores, cenozoico, reactivación.

\section{Abstract}

The Transmexican Volcanic Belt has been recognized as a major volcanic arc which crosses Mexico from the Pacific coast to the Gulf of Mexico. This volcanic arc had normal faulting and volcanism since Miocene. It has suggested that the volcanic arc is not parallel to the Mesoamerican trench because the subduction angle varies being gentler to the east. Also it has been proposed a preexisting zone of weakness below the Transmexican Volcanic Belt. In this work we present the deformation events that have been recorded north and south of the Transmexican Volcanic Belt in order to identify the time in which this zone began to be a crustal discontinuity and also to determine the deformation field precursor of the emplacement of the volcanic arc. We use as a reference the studies along the Taxco-San Miguel de Allende because it is the only recognized structure that crosses the Transmexican Volcanic Belt. In the Mesa Central, north of the Transmexican Volcanic Belt, the postlaramidic deformation occurred in three extensional events during the Eocene, Oligocene and Miocene to Recent. The three events liberated extension in two horizontal directions and shortening in the vertical direction. The Eocene event was extensional but the directions of the principal extensions are not very well known. The Oligocene event was the most important; it extended $20 \%$ in the $\sim E N E$-WSW direction. This event migrated toward west, finishing in the opening of the California Gulf, and also migrated toward south-southeast to the Transmexican Volcanic Belt. The most recent event, active since middle Miocene, has been developed in the Transmexican Volcanic Belt and its northern boundary. In the Sierra Madre Oriental, east of the Mesa Central and north of the Transmexican Volcanic Belt, the Cenozoic deformation has been minimum. South of the Transmexican Volcanic Belt, in the Taxco region, there were two postlaramidic deformation events, mainly liberated by NW-SE and N-S lateral faults. The first one occurred in late Eocene with a NNW-SSE horizontal extension direction and shortening to the ENE-WSW. The second event was early Oligocene with a maximum extension to the NE-SW and shortening to the NW-SE, both migrated toward east.

Most of the volcanic rocks along the Transmexican Volcanic Belt are Miocene, however it has reported Oligocene mafic volcanic rocks in the middle of the Mexico basin. Besides, this volcanic arc is located into tectonic basins bounded by major faults. In the case of the Mexico basin, faulting initiated in late Eocene time. It is concluded that since the Eocene, the deformation style was different in the Mesa Central and in the Sierra Madre del Sur, this implies the presence of a detachment zone between these provinces. From the Oligocene the lateral faults activity in the Sierra Madre del Sur produced shortening in the NW-SE principal direction, generating an oblique-extension with a left-lateral component within the zone now occupied by the central part of the Transmexican Volcanic Belt. The pre-Miocene deformation events formed a detachment zone in which the Mexico basin was developed, also through this zone was canalized the magmas surface forming the central part of the Transmexican Volcanic Belt.

Key words: Transmexican Volcanic Belt, major fault, Cenozoic, reactivation.

\section{Introducción}

Desde tiempos de Humboldt se reconoció en el centro de México un cinturón compuesto por numerosos volcanes con actividad reciente, que cruza desde Tepic hasta Veracruz, al que se le conoce actualmente como Faja Volcánica Transmexicana (FVTM). Entre las ideas sobre su origen resalta la que considera que se trata de una zona de debilidad antigua sobre la cual ha ascendido el magma para formar el arco volcánico actual; esta suposición se debe principalmente a su ubicación oblicua con respecto a la Trinchera de Mesoamérica (Mooser, 1972) (Figura 1).
Durante finales de la década de los 70 y principios de los 80 se publicaron varios modelos de la apertura y evolución del Golfo de México. Para acomodar geométricamente los bloques litosféricos se propuso la existencia de grandes zonas de cizalla ubicadas a lo largo y transversalmente a México y cuya actividad debió ocurrir entre 180 y $140 \mathrm{Ma}$. Entre esas grandes zonas de cizalla se ubicó una en el sitio que ocupa actualmente la FVTM (Anderson y Silver, 1974; Pindell, 1985; Ross y Scotese, 1988). La idea de una zona de cizallamiento de escala cortical o litosférica ya había sido insinuada por Mooser (1972) cuando indicó que la FVTM era una zona de sutura prepaleozoica reactivada 
en el terciario. La Faja Volcánica Transmexicana ha sido objeto de estudio en numerosos trabajos, en especial acerca del origen de los magmas y características del volcanismo; sin embargo la idea de que ha sido una zona de debilidad cortical antigua sigue siendo un tema no aclarado.

Se ha documentado que la cuenca de México (VenegasSalgado et al., 1985; Pérez-Cruz, 1988; Silva-Romo et al., 2002, Siebe et al., 2004), el graben San Pedro-Ceboruco (Ferrari et al., 2003a), el Bajío (Alaniz-Alvarez et al., 2001) y otras cuencas ubicadas a lo largo de la FVTM están limitadas por fallas mayores y alcanzan profundidades de varios cientos y hasta miles de metros. Si la FVTM está ubicada sobre una o varias depresiones tectónicas, resulta de primordial importancia determinar cuál fue el campo de deformación que las generó. Centraremos la discusión en el estilo y edad de los eventos de deformación que afectaron a los bloques adyacentes a la FVTM en su parte central, apoyándonos en los estudios que hemos realizado a lo largo del sistema de fallas Taxco-San Miguel de Allende, el cual cruza la FVTM de norte a sur y ha sido considerada una zona que limita bloques corticales (Nieto-Samaniego et al., 1999; Alaniz-Alvarez et al., 2002a). En vista de que el tren estructural de la FVTM es $c a$. E-W (Figura 1), analizaremos primero algunos rasgos con orientación transversal para poder evaluar la continuidad de rasgos geológicos en los bloques ubicados al norte y sur del arco volcánico. Posteriormente se describirán los distintos eventos de deformación regional en la parte central de México. Con estos elementos se definirá el carácter de frontera tectónica de la FVTM. Nuestro punto de partida es el Albiano, ya que se considera que en ese tiempo los basamentos premesozoicos de los bloques corticales de México ya ocupaban su sitio actual y no han tenido desplazamientos mayores desde entonces (Morán-Zenteno et al., 1988).

\section{Marco geológico}

En la parte central de México se unen varias provincias fisiográficas, destacan la Sierra Madre Occidental, la Mesa Central, la Sierra Madre Oriental, la Faja Volcánica Transmexicana, y la Sierra Madre del Sur (Raiz, 1959) (Figura 1). En este trabajo nos enfocaremos únicamente a algunos sectores de estas provincias cuya constitución geológica permite diferenciarlas.

\subsection{Elementos localizados al norte de la Faja Volcánica} Transmexicana:

La Sierra Madre Occidental es una provincia volcánica compuesta principalmente por ignimbritas silícicas emplazadas durante el Oligoceno-Mioceno en la parte occidental de México. Tiene una longitud superior a $1200 \mathrm{~km}$ abarcando desde la frontera de México con Estados Unidos hasta la región de Jalisco (Aranda-Gómez et al., 2000; Ferrari et al., 2005). En la Mesa Central ese volcanismo fue emplazado durante el Oligoceno, principalmente a manera de domos riolíticos, los cuales cubren un área superior a $10,000 \mathrm{~km}^{2}$ (Orozco-Esquivel et al., 2002). El basamento mesozoico que subyace al volcanismo silícico en la Mesa Central está compuesto principalmente por rocas marinas depositadas en un ambiente de cuenca, formando lo que se ha denominado el Geosinclinal Mexicano (Imlay, 1938), o la Cuenca Mesozoica del Centro de México (CarrilloBravo, 1971). Hacia el límite entre la Mesa Central y la Sierra Madre Occidental, el Mesozoico está formado por una secuencia volcanosedimentaria de edad Jurásico Tardío - Cretácico temprano (Martínez-Reyes, 1992; Quintero-Legorreta, 1992; Centeno-García y Silva Romo, 1997; Freydier et al., 2000) cuyos afloramientos siguen aproximadamente el límite entre la Sierra Madre Occidental y la Mesa Central.

La Sierra Madre Oriental es una cordillera constituida principalmente por rocas sedimentarias marinas mesozoicas que fueron deformadas por acortamiento durante la orogenia Laramide y que abarca prácticamente toda la parte oriental de México, desde Parral, Chih., hasta Tuxtepec, Oax. (Eguiluz et al., 2000). Hay numerosos trabajos que describen la estratigrafía de la Sierra Madre Oriental; según Eguiluz et al. (2000) el depósito de las rocas sedimentarias marinas inició en el Triásico Tardío, pero se mantuvo constante únicamente desde el Jurásico

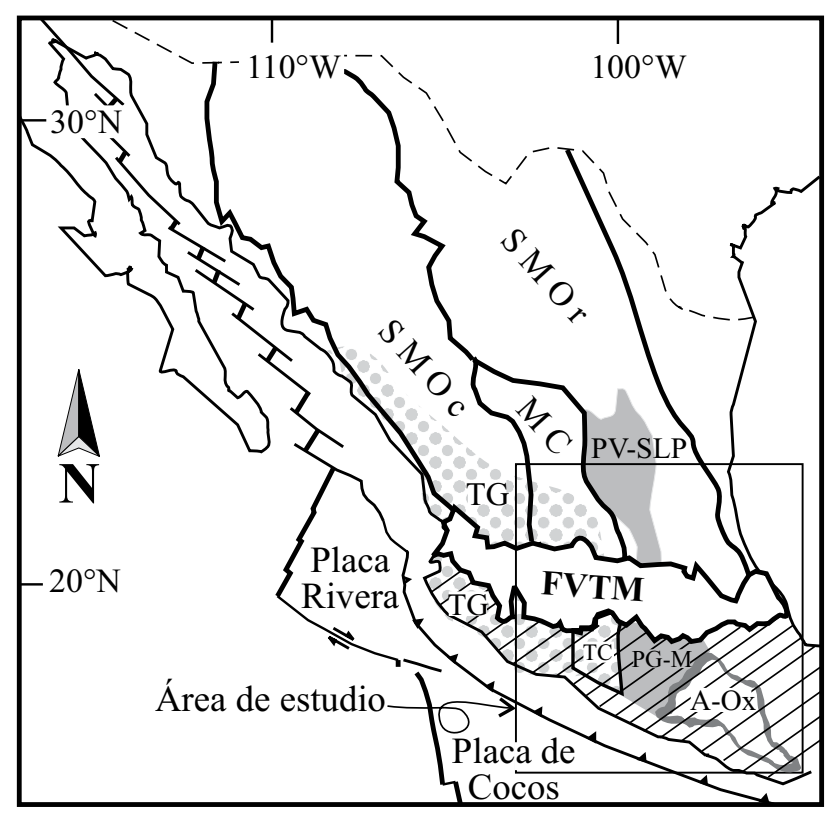

Figura 1. Localización del área de estudio. SMOc: Provincia volcánica Sierra Madre Occidental, SMOr: Sierra Madre Oriental, FVTM: Faja Volcánica Transmexicana, MC: Mesa Central, PG-M: Plataforma Guerrero-Morelos, TC: Complejo Metamórfico Tierra Caliente, TG: arcos volcánicos mesozoicos del terreno Guerrero, A-Ox: bloque que contiene los basamentos de los complejos Acatlán y Oaxaqueño, PV-SLP: Plataforma Valles-San Luis Potosí, líneas diagonales: Sierra Madre del Sur, trama punteada: terreno Guerrero. 
Superior hasta el Cretácico Superior con una distribución de facies muy variable. La plataforma Valles-San Luis Potosí es uno de los elementos paleogeográficos de esta cordillera (Figura 1), se distingue por su cubierta delgada de sedimentos marinos del Jurásico Superior que yacen bajo una secuencia de hasta $4000 \mathrm{~m}$ de rocas evaporíticas y calizas de tipo arrecifal y postarrecifal del Cretácico Inferior y Superior (Carrillo-Bravo, 1971). La orogenia Laramide produjo pliegues y cabalgaduras con vergencia hacia el este-noreste, su actividad abarca desde fines del Cretácico al Eoceno migrando el frente tectónico de oeste a este (Eguiluz et al., 2000).

Al norte de la Faja Volcánica Transmexicana se tiene, para el Jurásico Superior - Cretácico y de este a oeste, la siguiente distribución paleogeográfica: una plataforma localizada dentro de la Sierra Madre Oriental, de ella se pasa a una cuenca marina que se localiza en la Mesa Central, y más al oeste, en el límite de la Mesa Central con la Sierra Madre Occidental, existía un ambiente de arcos volcánicos marinos.

\subsection{Elementos localizados al sur de la Faja Volcánica} Transmexicana:

La plataforma Guerrero-Morelos se ubica en la parte central de la Sierra Madre del Sur. En ella aflora una secuencia sedimentaria marina somera que abarca desde el Neocomiano hasta el Turoniano (Anhidrita Huitzuco, Formación Morelos y Formación Cuautla) (HernándezRomano et al., 1997; Aguilera-Franco, 2003), en la parte oriental y en la base aparecen conglomerados rojos continentales con horizontes de caliza intercalados (Formación Zicapa, De Cserna et al., 1980). Las rocas de la plataforma Guerrero-Morelos presentan estructuras de deformación contráctil con vergencia hacia el este formadas a fines del Cretácico, cuya edad de deformación también muestra una migración hacia el este. También han sido reportadas estructuras que indican vergencia hacia el poniente en las inmediaciones de las cabalgaduras de Teloloapan (Salinas-Prieto et al., 2000) y Papalutla (Cerca et al., 2004). Aunque no se ha considerado estrictamente que la plataforma Guerrero-Morelos sea parte de la Sierra Madre Oriental, la presencia de esta plataforma justo al sur de la plataforma Valles-San Luis Potosí, y el hecho de estar los afloramientos de ambas separadas por la Faja Volcánica Transmexicana de edad Cenozoica, indica continuidad en la paleogeografía del borde de las plataformas continentales para el Cretácico Temprano (Figura 1).

El Complejo Metamórfico Tierra Caliente está ubicado al poniente de la plataforma Morelos-Guerrero, entre Arcelia y la cabalgadura de Teloloapan (Figura 2). Se caracteriza por ser una secuencia volcanosedimentaria cretácica marina metamorfizada en bajo grado (OrtegaGutierrez, 1981); esta secuencia contiene andesitas, lavas almohadilladas, ignimbritas, tobas, areniscas y calizas que forman parte de las unidades Roca Verde Taxco Viejo, Esquisto Taxco, filita Almoloya (Cabral-Cano et al., 2000). El Complejo Tierra Caliente forma parte de lo que se ha denominado terreno Guerrero (Campa y Coney, 1983). En este trabajo nos centraremos únicamente en su carácter predominantemente volcánico que contrasta con el predominio de sedimentos marinos sin contenido volcánico de la plataforma Guerrero-Morelos.

Podemos deducir que a los lados del SFTSMA, al sur de la Faja Volcánica Transmexicana, se tiene para el Cretácico Inferior, de este a oeste, una plataforma, de la cual se pasa a un ambiente de arcos volcánicos marinos. A diferencia de esto, en la región localizada al norte de la Faja Volcánica Transmexicana existe una cuenca marina que separa esos dos paleoambientes.

La provincia magmática de la Sierra Madre del Sur está constituida por rocas volcánicas y plutónicas terciarias expuestas en una amplia región al sur de la Faja Volcánica Transmexicana, las cuales fueron emplazadas desde el Paleógeno hasta el Mioceno (Morán-Zenteno et al., 2000). Las rocas plutónicas, principalmente graníticas, afloran en una franja paralela a la margen costera del Océano Pacífico; mientras que las rocas volcánicas se emplazaron en la parte interna de la Sierra Madre del Sur. Varios tipos de basamento subyacen esta provincia, el terreno Guerrero al occidente y los complejos Acatlán y Oaxaqueño al oriente de la plataforma Guerrero-Morelos.

\section{Edad de la orogenia Laramide}

Se sabe que, en general, la orogenia Laramide migró de oeste a este en la región que abarca este estudio (De Cserna et al., 1980, Eguiluz et al., 2000). Para estimar la edad de la deformación en una localidad determinada se consideró la edad reportada de la roca más joven afectada y la más antigua no afectada por ella. Se compara la edad de la deformación en las plataformas Valles-San Luis Potosí y Guerrero-Morelos.

En la sierra de Guanajuato $\left(101^{\circ}-102^{\circ} \mathrm{W}\right)$ la edad de la deformación laramídica se establece entre el Cenomaniano temprano, edad máxima de la Caliza La Perlita, la cual está ligeramente plegada, y 54 - $58 \mathrm{Ma}$, edad del Granito Comanja sin deformar (Quintero-Legorreta, 1992). Estas edades son consistentes con la edad de la primera deformación extensional postlaramídica en la sierra de Guanajuato, la cual se fechó con la edad de un derrame de lava de $49 \mathrm{Ma}$ intercalado en el Conglomerado Guanajuato (Aranda-Gómez y McDowell, 1998). En la parte sur de la plataforma Valles-San Luis Potosí, la edad de la deformación está constreñida por la Formación Cárdenas (Campaniano-Maastrichtiano, Carrillo-Bravo, 1971), la cual contiene las rocas más jóvenes deformadas contráctilmente y $43 \mathrm{Ma}$, que es la edad reportada del tronco granítico postectónico más antiguo emplazado sobre los sedimentos plegados (Vassallo et al., 2004). 


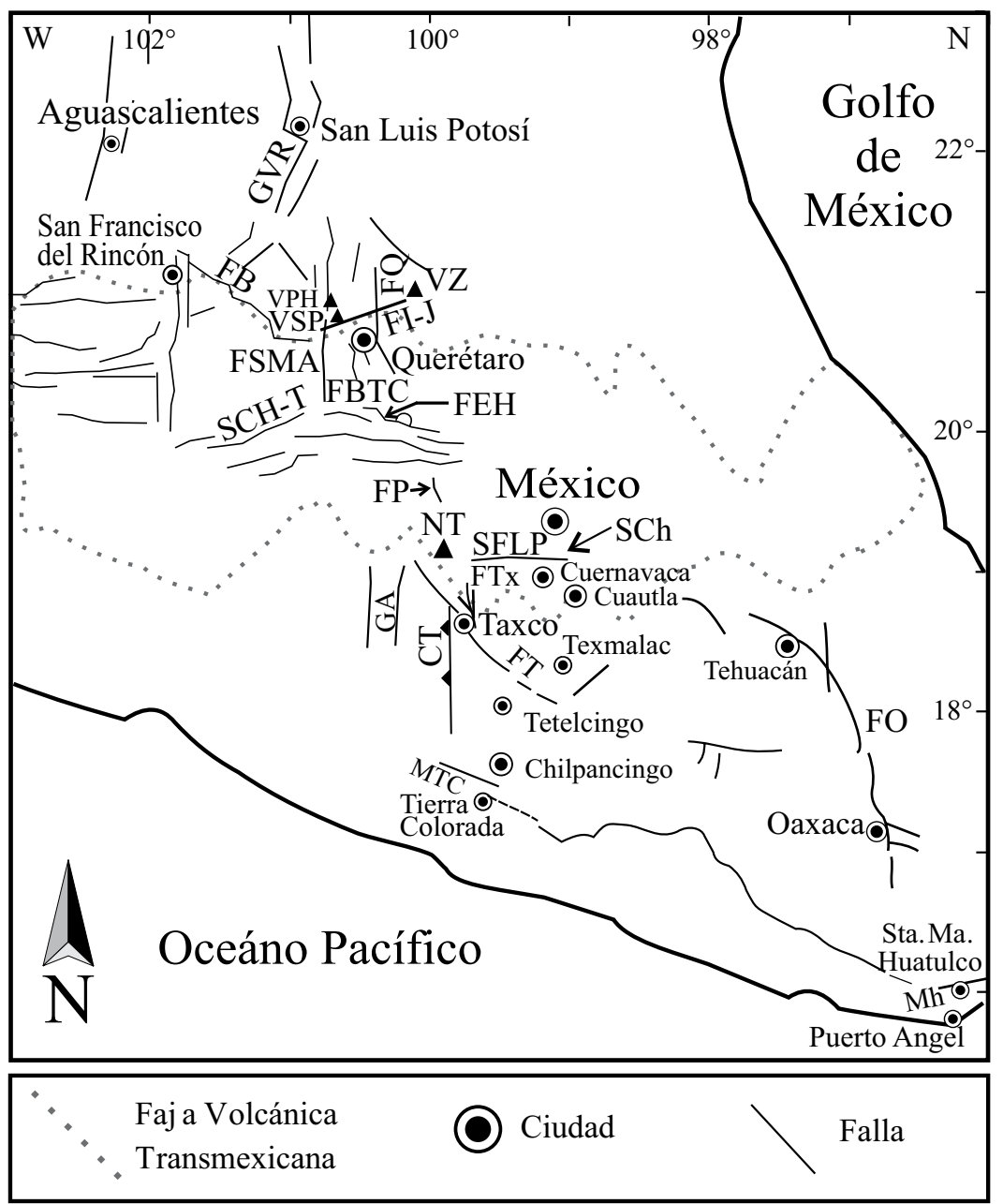

Figura 2. Ubicación de las fallas mayores, volcanes y ciudades referidas en el texto. GVR: graben Villa de Reyes; FQ: falla Querétaro; FBTC: fallas Bartolomé, Tlacote, La Cuesta y Lagunillas-Huimilpan; FB: falla del Bajío; FI-J: fallas Ixtla-La Joya; FSMA: falla San Miguel de Allende; SCH-T: sistema Chapala-Tula; FT: falla Tetipac, Chichila y Amates; FLP: sistema de fallas La Pera; FTx: fallas Taxco, San Gregorio, Coapango y Acamixtla; FEH: falla Epitacio Huerta; FP: falla Perales; GA: graben de Arcelia; CT: cabalgadura Teloloapan; FO: Falla de Oaxaca; MTC: milonitas de Tierra Colorada; Mh: milonitas de Huatulco; SCh: Sierra de Chichinautzin; VZ: Volcán Zamorano; VPH: Volcán Palo Huérfano; VSP: Volcán San Pedro; NT: Volcán Nevado de Toluca.

En la plataforma Guerrero-Morelos, en Tetelcingo $\left(99^{\circ} 35^{\prime} \mathrm{W}\right)$, localizado al sur de la plataforma Valles-San Luis Potosí, la edad de la orogenia Laramide se estableció entre el Turoniano-Coniaciano, por el contenido fósil de la Formación Mexcala que es la roca más joven deformada contráctilmente (Ortega-Gutiérrez, 1980) y el Maastrichtiano por la edad de la Formación Tetelcingo (66 $\pm 2.3-68.8 \pm 2.4 \mathrm{Ma}$, edad K-Ar biotita y roca entera en basalto, Ortega-Gutiérrez, 1980). Mientras que en la parte oriental de esta plataforma, en Texmalac, la edad máxima de la deformación es posterior al Maastrichtiano ya que la Formación Mexcala en ese lugar contiene fósiles del Maastrichtiano medio-tardío (Alencaster, 1980).

Los rangos de edad obtenidos para la orogenia Laramide nos permiten establecer que la migración del frente tectónico hacia el oriente y ocurrió de manera semejante en las plataformas que se encuentran al norte y al sur de la FVTM.

\section{Sistema de fallas Taxco-San Miguel de Allende}

Demant (1978) nombró "lineamiento Taxco-San Miguel de Allende" a un rasgo estructural que cruza la FVTM con una orientación NNW-SSE. Él propuso que esta estructura tuvo un movimiento lateral derecho durante el Mioceno, basado en el desplazamiento aparente de la FVTM al ser cruzada por esta estructura. En la última década se han aportado una gran cantidad de datos sobre este lineamiento y no obstante que se prolonga más allá de los sitios que originalmente se consideró, se decidió mantener Taxco y San Miguel de Allende en el nombre de esta estructura por lo conocida que es en la comunidad geológica mexicana. De esta manera, se le llama sistema de fallas Taxco-San Miguel de Allende (SFTSMA) al conjunto de fallas con orientación $c a$. N-S, NNW-SSE que atraviesan el centro de México y que divide bloques corticales con diferentes historias geológicas y espesores de la corteza y topografía 
distintos (Alaniz-Alvarez et al., 2002a). Su principal manifestación geofísica es una anomalía gravimétrica significativa (Urrutia-Fucugauchi y Flores-Ruiz, 1996).

Estudios recientes sobre el SFTSMA muestran que su longitud es mayor que $450 \mathrm{~km}$, abarcando desde más al norte de la sierra de Catorce, S.L.P. hasta el estado de Guerrero, y llega a tener hasta $30 \mathrm{~km}$ de ancho en la región de Querétaro (Alaniz-Alvarez et al., 2002a) (Figura 2). El SFTSMA incluye fallas con longitudes que van de 15 a $50 \mathrm{~km}$. La cinemática, desplazamiento y edad de las fallas mayores de este sistema han sido cuidadosamente documentadas por diversos autores: el graben de Villa de Reyes por Tristán-González (1986), la falla San Miguel de Allende en la Mesa Central por Nieto-Samaniego y Alaniz-Alvarez (1994); las fallas San Bartolomé, Tlacote y Querétaro, en la transición de la Mesa Central y la FVTM por Alaniz-Alvarez et al. (2001); las fallas La Cuesta y Lagunillas - Huimilpan por Dávalos-Alvarez (2004), las fallas Epitacio Huerta y Perales en la FVTM por Suter et al. (1995); las fallas norte-sur en El Oro-Tlalpujahua por Flores (1920), el sistema de pilares y fosas tectónicas al sur del Nevado de Toluca en la parte meridional de la FVTM por García-Palomo et al. (2000); y las fallas Taxco, San Gregorio, Coapango y Acamixtla por Alaniz-Alvarez et al. (2002b) y la cabalgadura Teloloapan por Cabral-Cano et al. (2000) en la parte septentrional-central de la Sierra Madre del Sur. La traza de la estructura es N-S fuera de la FVTM y cambia a una dirección NNW-SSE dentro de ella.

Dentro de la Faja Volcánica Transmexicana la presencia del SFTSMA es evidenciada por fallas normales transversales al sistema principal de fallas Chapala-Tula en las regiones de Querétaro, Acambay, El Oro y Toluca, y por el alineamiento de 12 volcanes poligenéticos en la traza de esta estructura (Alaniz-Alvarez et al., 1998). Este sistema continúa hacia el norte de la ciudad de San Luis Potosí formando el límite de una gran depresión tectónica que constituye la prolongación norte del graben de Villa de Reyes hasta el norte de la sierra de Catorce (MoreiraRivera et al., 1996). Hacia el sur, el límite poniente de la plataforma Guerrero-Morelos lo constituye una serie de fallas N-S a las que pertenece la cabalgadura Teloloapan, las cuales limitan la secuencia volcanosedimentaria marina mesozoica del Complejo Metamórfico Tierra Caliente, poniéndola en contacto con la secuencia mesozoica marina carente de material volcánico de la plataforma GuerreroMorelos (Cabral-Cano et al., 2000; Salinas-Prieto et al. 2000) (Figura 1, 2).

La cinemática y edad de las fallas del SFTSMA han obedecido a la deformación de la provincia a la que pertenecen, así en la Mesa Central y en la FVTM la componente principal de movimiento ha sido normal y ha ocurrido en dos tiempos distintos, en el Oligoceno y en el Mioceno- Reciente (Alaniz-Alvarez et al., 2002a), mientras que en la región de Taxco la componente principal fue lateral, con sentidos tanto derecho como izquierdo, ya que estuvieron sujetas a dos fases de deformación distintas ocurridas en el Eoceno tardío y Oligoceno temprano (Alaniz-Alvarez et al., 2002b).

El ancho del SFTSMA es variable, dependiendo del número de fallas que acomodan la deformación. En la región de Querétaro la zona de falla tiene su máximo ancho con cerca de $30 \mathrm{~km}$, abarca desde la falla San Miguel de Allende hasta la falla Querétaro, mientras que en las otras localidades es más angosto.

\subsection{El sistema de fallas Taxco-San Miguel de Allende} como límite de elementos paleogeográficos

Se ha mencionado que el SFTSMA se encuentra en el límite entre dos bloques corticales con distinta topografía, espesor de la corteza y distintas características geológicas (Alaniz-Alvarez et al. 2002a). Regionalmente, está entre la Mesa Central y la Sierra Madre Oriental; entre dos sectores de la FVTM con distintas características estructurales y tipo de volcanismo, y entre el Complejo Metamórfico Tierra Caliente y la plataforma Guerrero-Morelos. Hay un elemento paleogeográfico mayor de edad mesozoica que se localiza bajo el SFTSMA, el cual es el límite occidental de las plataformas marinas Valles San Luis Potosí y Guerrero-Morelos. El límite de afloramientos de las secuencias volcanosedimentarias marinas que coincide con el SFTSMA se encuentra únicamente desde San Miguel de Allende hasta el sur de Teloloapan. En San Miguel de Allende se encuentra el contacto entre la Formación Soyatal, del Turoniano Medio (HernándezJáuregui et al., 2000) y la secuencia volcanosedimentaria; sin embargo el volcanosedimentario se encuentra aflorando nuevamente al oriente en la sierra de los Cuarzos, localizada al norte de la ciudad de Querétaro, entre las fallas San Miguel de Allende y Querétaro (Alaniz-Alvarez et al., 2001). A partir de la sierra de los Cuarzos, $20 \mathrm{~km}$ al norte de la ciudad de Querétaro, los afloramientos de las rocas volcanosedimentarias mesozoicas se desvían hacia el noroeste (Centeno-García y Silva-Romo, 1997). En la región de El Oro-Tlalpujahua, Mich. afloran las rocas volcanosedimentarias marinas mesozoicas (Flores, 1920; Pasquaré et al., 1991) en el lado poniente de la traza del SFTSMA, siendo éste el único afloramiento del basamento dentro de la FVTM. Al sur de la FVTM, Cabral-Cano et al. (2000) presentan la historia de deformación del Complejo Metamórfico Tierra Caliente, entre Arcelia y Teloloapan, para determinar la naturaleza de su límite oriental con la plataforma Guerrero-Morelos. Ellos concluyen que el contacto forma parte de una serie de cabalgaduras de rumbo N-S generadas durante una deformación contractiva ocurrida a fines del Cretácico y por lo tanto correlacionable con la orogenia Laramide. La cabalgadura de Teloloapan no presenta rasgos de una deformación mayor, como pudiera ser la presencia de metamorfismo de alta presión o bien de intensa deformación frágil. Cabral-Cano et al. (2000) concluyen que la cabalgadura de Teloloapan no es 
un límite entre bloques corticales sino la manifestación de un relieve antiguo sujeto a deformación compresiva. El límite poniente de las plataformas continentales VallesSan Luis Potosí y Guerrero-Morelos coincide con la traza del SFTSMA desde la sierra de Catorce hasta el sur de Teloloapan. Por lo tanto, la traza norte-sur del SFTSMA se localiza a lo largo de la transición entre una zona de plataforma continental y una de mayor batimetría; el cambio de pendiente y las diferencias en espesores corticales existentes para el Cretácico son el origen que nosotros proponemos para la discontinuidad con orientación norte-sur noroeste-sureste que controló la localización del fallamiento del SFTSMA durante el cenozoico.

Lo anterior explica algunos rasgos que se presentan sobre este lineamiento: la persistencia de fallas con orientación NNW-SSE aún cuando existan sistemas de fallas con otras orientaciones; la anomalía gravimétrica que indica distintos espesores de corteza a los lados del SFTSMA; el ensanchamiento de la zona de falla del SFTSMA entre San Miguel de Allende y Querétaro donde la plataforma Valles-San Luis se estrecha; la ubicación del límite oriental del terreno Guerrero en el sur y la continuación de la traza del SFTSMA al norte de la ciudad de San Luis Potosí sobre el límite entre la plataforma Valles-San Luis Potosí y la Cuenca Mesozoica del Centro de México.

\section{Eventos de deformación cenozoicos}

Hay pocos estudios del sur de México enfocados a las deformaciones cenozoicas y prácticamente todos están concentrados al oriente del SFTSMA, con excepción de los estudios en los grábenes de Colima y Arcelia que están al poniente de esta estructura. En la Mesa Central y la parte sur de la Sierra Madre Occidental la deformación cenozoica está bien documentada (e.g., Nieto-Samaniego et al., 1999), mientras que en la zona localizada al oriente del SFTSMA, en la Sierra Madre Oriental, se tiene muy poca información y al parecer existe poca o mínima deformación cenozoica.

\subsection{Eventos paleocénicos}

Probablemente uno de los aspectos menos estudiados de la región que abarca este estudio es la deformación paleocénica, la cual incluye las fases terminales de la orogenia Laramide y la registrada con el depósito de lechos rojos asociados a fallas laterales o normales.

Aunque no es posible inferir diferencias entre la deformación paleocénica al norte y sur de la Faja Volcánica Transmexicana, consideramos necesario mencionar algunos datos publicados sobre posibles eventos de deformación durante esa época. En la parte central de la Sierra Madre del Sur se ha propuesto un evento de deformación posterior al clímax contráctil laramídico, ese evento produjo pliegues amplios y cabalgaduras con vergencia hacia el poniente. Salinas-Prieto et al. (2000) y Cabral-Cano et al. (2000) proponen que estas estructuras afectan tanto a la secuencia volcanosedimentaria de Arcelia como a la plataforma Guerrero-Morelos, y que están mucho menos desarrolladas que las estructuras con vergencia hacia el oriente. En ambos trabajos se propone que esta fase se desarrolló probablemente por el colapso gravitacional de la pila de sedimentos acumulada durante el acortamiento de la orogenia Laramide. Cerca-Martínez (2004) reportó también una serie de pliegues amplios y cabalgaduras con vergencias opuestas y posteriores a las estructuras generadas por la orogenia Laramide en el extremo oriental de la plataforma Guerrero-Morelos. Él documentó una serie de cuencas desarrolladas durante el terciario temprano las cuales estarían asociadas a estas estructuras y declara que la generación de estas estructuras se debe a un evento transpresivo posterior a la orogenia.

La edad de las estructuras contractivas con vergencia hacia el poniente únicamente afectaron algunas porciones del sur de México, su edad y cinemática están poco estudiadas y no existe un consenso acerca de su origen. Las evidencias que se tienen indican que se formaron inmediatamente después del pico de la deformación laramídica (Cerca-Martínez, 2004; Cabral-Cano et al., 2000; Salinas-Prieto et al., 2000), lo que abre la posibilidad de que sea un evento tardío de esa orogenia, ocurrido en el Paleoceno.

El depósito de lechos rojos postlaramídicos ocurrió tanto en la Mesa Central como en la Sierra Madre del Sur, estos depósitos fueron reconocidos por Edwards (1955) como conglomerados continentales postorogénicos. Estudios en la sierra de Guanajuato y en Taxco muestran que estos depósitos son de edad Paleoceno-Eoceno y no están relacionados con el evento de deformación contractiva paleocénica. Aranda-Gómez y McDowell (1998) le asignaron al Conglomerado de Guanajuato, localizado en la Mesa Central, una edad eocénica y lo consideran formado sintectónicamente con un evento de extensión. En el sur de México, el Conglomerado Rojo de Taxco subyace a la Ignimbrita Acamixtla de 38 Ma y está limitado por fallas con dirección NW-SE (Alaniz-Alvarez et al., 2002b). Aunque es posible que estos depósitos se originaran asociados a un evento de deformación extensional Paleoceno-Eoceno de manera similar a lo documentado en Guanajuato, no se cuenta con información suficiente para documentarlo.

\subsection{Eventos eocénicos}

El primer evento de extensión postlaramídico ocurrió durante gran parte del Eoceno en la Mesa Central, mientras que al sur de la FVTM, durante el Eoceno tardío se liberó deformación transcurrente (Figura 3).

Para la Mesa Central, Aranda-Gómez y McDowell 
(1998) documentan un evento de extensión posterior a los 49 y anterior a los $37 \mathrm{Ma}$. Las direcciones de extensión no están establecidas ya que las fallas NW y NE que liberaron la deformación fueron reactivadas posteriormente durante el Oligoceno en un evento mucho mayor. Aranda-Gómez y McDowell (1998) proponen que el Conglomerado Guanajuato registró dos fases de extensión eocénica, la primera activó fallas NW con extensión hacia el NE-SW y la segunda activó fallas NE con una extensión hacia el NW-SE.

En la parte oriental de la Sierra Madre del Sur, la deformación eocénica se liberó a través de fallas laterales izquierdas con orientación NW, paralelas a la costa (Figura 3). Este evento se registró desde cerca de la margen pacífica, en rocas miloníticas, hasta el borde sur de la Faja Volcánica Transmexicana en la región de Taxco, en fallas frágiles. Las milonitas de Tierra Colorada generadas con esta cinemática se han relacionado con el desplazamiento del bloque de Chortis hacia el sureste (Schaaf et al., 1995). En Taxco, las fallas de Tetipac y Chichila, con orientación $\mathrm{N} 45^{\circ} \mathrm{W}$ y verticales, tuvieron desplazamiento izquierdo entre $36 \mathrm{y}$ 33 Ma (Alaniz-Alvarez et al., 2002b). Morán-Zenteno et al. (2004) reportan que sobre la falla de Amates, la cual tiene dirección $\mathrm{N} 45^{\circ} \mathrm{W}$ y es parte del sistema de fallas que incluye la falla de Tetipac, se emplazó un dique que contiene estrías e indicadores cinemáticos de movimiento lateral izquierdo; este dique contiene material que alimentó a la caldera de Tilzapotla de $34 \mathrm{Ma}$ de edad. En el sur de México se cuenta con edades similares para este evento, en localidades con la misma longitud oeste ubicadas desde la costa hasta el margen meridional de la FVTM: se tiene una edad >34 Ma (Riller et al., 1992) en Tierra Colorada y entre 38 y 33 Ma en Taxco (Alaniz-Alvarez et al., 2002b) y Tilzapotla; la dirección de máxima extensión estimada en Taxco es hacia el NNW-SSE (Figura 3, Alaniz-Alvarez et al., 2002b, figura 6a).

\subsection{Eventos oligocénicos}

Durante el Oligoceno (de 32 a $24 \mathrm{Ma}$ ) se registró deformación extensional en la Mesa Central liberada a través de un complejo sistema de fallas normales con orientaciones principales NW-SE, NE-SW y N-S. Esta deformación fue la principal registrada en esa región y tuvo una extensión del $20 \%$ con dirección $\mathrm{N} 79^{\circ} \mathrm{E}$ y $11 \%$ con dirección $\mathrm{N} 11^{\circ} \mathrm{W}$ (Nieto-Samaniego et al., 1999). En la región de San Miguel de Allende se documentó que los tres sistemas de fallas estuvieron activos durante el evento oligocénico, las fallas principales de estos sistemas son la falla del Bajío, el graben de Villa de Reyes y la falla San Miguel de Allende (Alaniz-Alvarez et al., 2001).

En el sur, en la región de Taxco se registró un evento de deformación transcurrente durante el Oligoceno reactivando las fallas preexistentes (Alaniz-Alvarez et al., 2002b). Las fallas N-S tuvieron desplazamiento lateral izquierdo mientras que las fallas de Tetipac y Chichila con rumbo $\mathrm{N} 45^{\circ} \mathrm{W}$ tuvieron desplazamiento lateral derecho. La dirección del máximo alargamiento horizontal es hacia el NE-SW, mientras que la de máximo acortamiento horizontal es hacia el NW-SE (Figura 3); es notable que las direcciones principales de deformación cambiaron en un lapso corto en esta región entre el final del Eoceno y el inicio del Oligoceno.

\subsection{Evento del Mioceno al Reciente}

Del Mioceno al Reciente la deformación en la parte central de México se ha concentrado a lo largo de la FVTM y se ha liberado a través de fallas normales. Martínez-Reyes y Nieto-Samaniego (1990) cartografiaron las fallas que se encuentran al lado poniente del SFTSMA en la Faja volcánica Transmexicana, las que en conjunto Suter et al. (1992, 1995) denominaron sistema de fallas Chapala-Tula (Figura 2). Las fallas de este sistema son normales con una componente lateral izquierda subordinada. Suter et al., (2001) calcularon para el sistema Chapala-Tula una extensión menor que 3\% hacia el NNW-SSE durante el cuaternario.

Al oriente del SFTSMA, en la región de Apan, al norte de la $\mathrm{Cd}$. de México, un sistema de pilares y fosas tectónicas se formaron con rumbo NNE-SSW afectando rocas volcánicas del Mioceno medio al Holoceno, pudiéndose inferir para ese lapso una dirección de extensión WNWESE (García-Palomo et al., 2002).

El límite entre la FVTM y la Mesa Central está marcado por el afloramiento del basamento mesozoico a lo largo de las fallas del Bajío e Ixtla-La Joya. En estas fallas se ha documentado actividad durante los eventos oligocénico y miocénico (Alaniz-Alvarez et al., 2002a), el primero predominó en la Mesa Central, mientras que el segundo en la FVTM. En la margen septentrional de la parte central de la FVTM coinciden dos sistemas de fallas, las que pertenecen al sistema Chapala-Tula de dirección WSW-ENE, y las del sistema Taxco-San Miguel de Allende con orientación NNW-SSE. En la región entre Celaya y Querétaro, Alaniz-Alvarez et al. (2002a) documentaron que los tres sistemas de fallas, con orientaciones N-S, NW-SE y NESW se activaron contemporáneamente durante los mismos eventos de deformación. Un estudio detallado hecho por Dávalos-Alvarez (2004) en la región de Huimilpan, a 30 $\mathrm{km}$ al sur de Querétaro, documentó que los tres sistemas de fallas se activaron durante el Plioceno en el mismo evento de deformación pero de manera alternada.

En la Sierra Madre del Sur, la deformación miocénica se registró en Oaxaca, donde ignimbritas de la Formación Suchilquitongo de 19 Ma están cortadas por fallas normales con rumbo $\mathrm{N} 15^{\circ} \mathrm{W}$, sugiriendo un evento de extensión hacia el ENE-WSW (Urrutia-Fucugauchi y FerrusquíaVillafranca, 2001). La cartografía geológica a detalle de la Mesa Central y la región de Querétaro han permitido 
a)
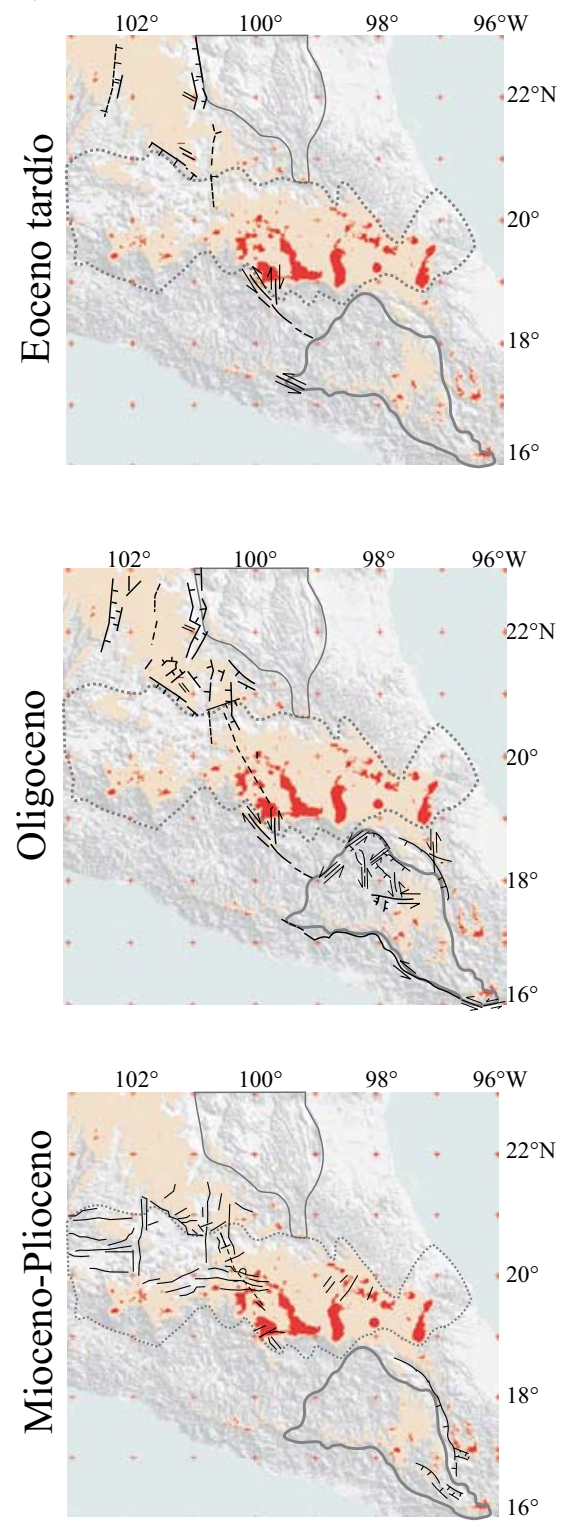

b)
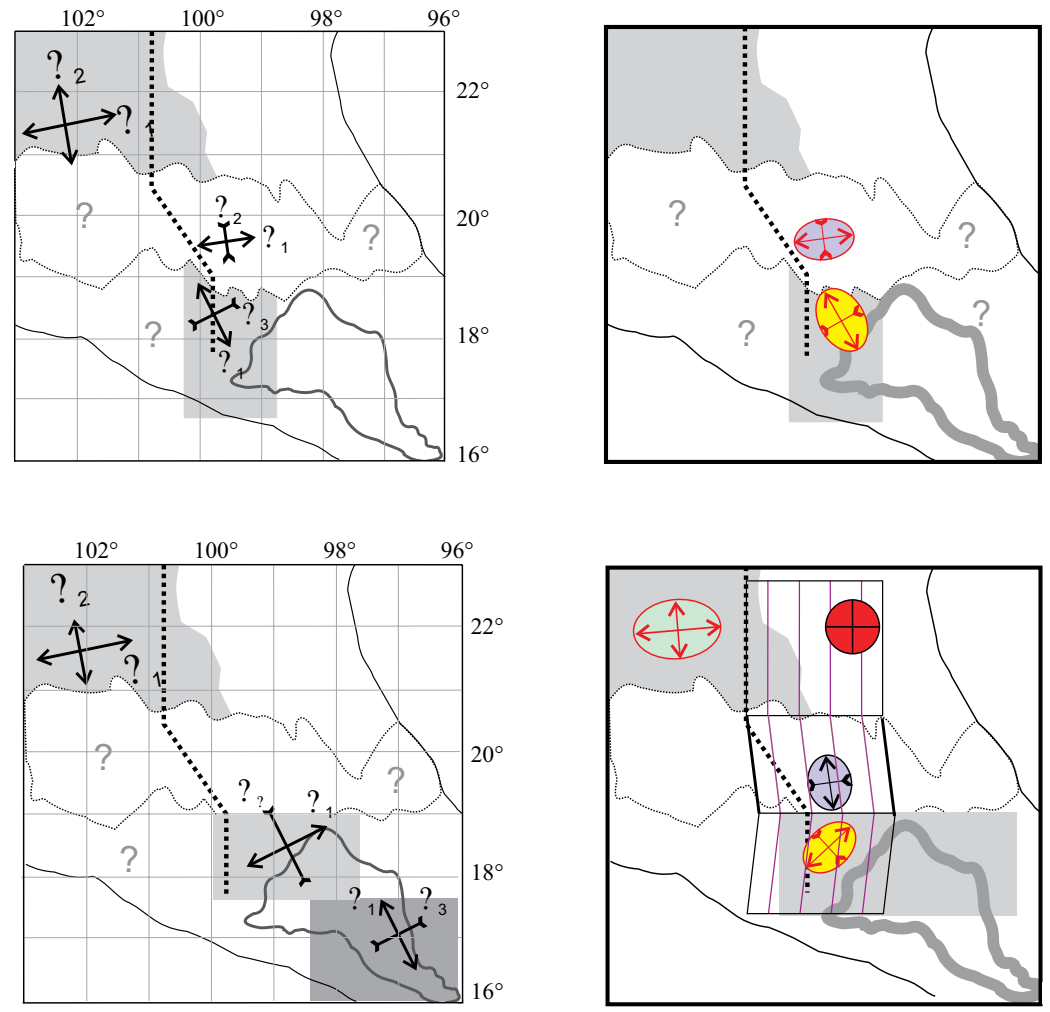

c)
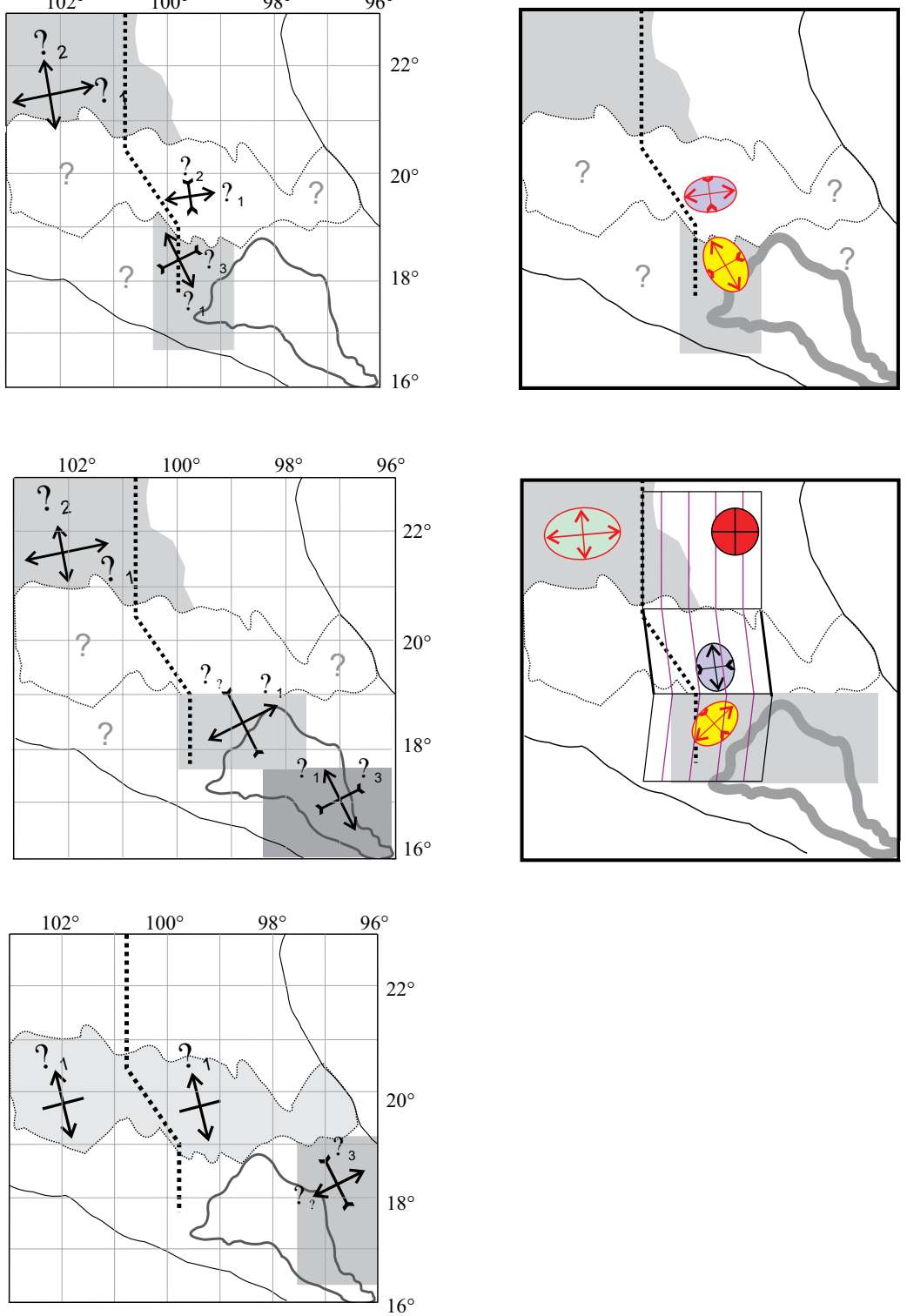

\begin{tabular}{|c|c|c|c|c|}
\hline$\lesssim$ & Falla lateral & & Zona deformada & Sin deformación \\
\hline & Falla normal & & Zona sin deformar & Plataforma Valles - San Luis Potosí \\
\hline & Direcciones aproximadas de & $?$ & Zona sin datos & Bloque Acatlán - Oaxaqueño \\
\hline 古 & $\begin{array}{l}\text { ejes principales horizontales } \\
\text { de deformación infinitesimal }\end{array}$ & $?_{1}$ & Extensión máxima & Faja Volcánica Transmexicana \\
\hline & $\begin{array}{l}\text { Elipse de deformación finita } \\
\text { horizontal }\end{array}$ & $\leftrightarrow{ }^{?_{2}}$ & $\begin{array}{l}\text { Extensión mínima } \\
\text { Contracción máxima }\end{array}$ & $\begin{array}{l}\text { Sistema de fallas } \\
\text { Taxco - San Miguel de Allende }\end{array}$ \\
\hline
\end{tabular}

Figura 3. Esquema mostrando los eventos de deformación principales. En la columna a) se muestra las fallas principales que tuvieron actividad en distintos tiempos. Las áreas en color beige y rojo tienen elevaciones mayores a 2000 y $3000 \mathrm{msnm}$, respectivamente; esas cotas destacan los límites de la Faja Volcánica Transmexicana y la Mesa Central. Se observa también distintas elevaciones a los lados de la falla Taxco-San Miguel de Allende, al norte de la FVTM las elevaciones mayores se localizan hacia el oeste, y al sur de la FVTM hacia el este. La columna b) muestra en sombreado las regiones que tuvieron deformación en distintos tiempos. Las flechas indican las direcciones de los ejes principales horizontales de deformación infinitesimal discutidos en el texto; dichos ejes se infirieron a partir de la orientación de las fallas que liberaron dicha deformación. En la columna c) se muestra la elipse de deformación finita inferida para la FVTM a partir de las deformaciones ocurridas al norte y sur de ella; se supone una deformación pequeña. Los signos de interrogación señalan sitios donde no se tiene información. 
reconocer la presencia de rocas volcánicas miocénicas cortadas por fallas normales con desplazamientos pequeños (Orozco-Esquivel et al., 2002). Lo anterior indica que la deformación miocénica existió tanto en la parte meridional de la Mesa Central como en Oaxaca, aunque la mayor parte de ella se concentró a lo largo de la FVTM.

\section{Fallas mayores que limitan la FVTM en su porción central}

La actividad de las fallas mayores tienen un papel muy importante en la deformación, ya que ellas no sólo tienen la capacidad de liberar una gran cantidad de desplazamiento, sino también tienen un alto potencial de reactivación y controlan el tipo de deformación reactivando o produciendo fallas de neoformación asociadas a ellas.

En la Mesa Central se ha observado que los tres sistemas de fallas principales son paralelos a las fallas mayores (Nieto-Samaniego et al., 1999), esto es evidente tanto para la deformación miocénica como para la oligocénica. Los modelos analógicos desarrollados por Dubois et al. (2002) indican que en una segunda fase de extensión sobrepuesta y oblicua a un conjunto de estructuras desarrolladas en una fase previa, se reactivarán las estructuras formadas en la primera fase y se forman nuevas fallas paralelas a éstas.

Una falla normal con más de $40 \mathrm{~km}$ de largo implica que su profundidad puede alcanzar el límite entre el régimen frágil y dúctil. En el área considerada en este estudio, las fallas con más de $40 \mathrm{~km}$ de largo, en la Mesa Central, son la falla del Bajío, la falla Ixtla-la Joya, el graben de Villa de Reyes (en su parte norte solamente), la falla San Miguel de Allende y la falla de Querétaro (Figura 2). Estas fallas constituyen el límite entre dominios estructurales y secuencias estratigráficas distintas.

En este trabajo solamente se describirán las fallas que se encuentran en los límites de la FVTM y se incluye al sistema de fallas La Pera que constituye el límite meridional de las cuencas de México y de Toluca. Se consideraron como parte del del límite estructural de la FVTM a las fallas que tienen las siguientes características: (1) exponen las rocas mesozoicas, (2) su traza puede seguirse por más de 40 $\mathrm{km}$, (3) han sido activadas en los eventos de deformación oligocénica y miocénica, (4) su desplazamiento ha sido calculado en más de $300 \mathrm{~m}$ y (5) su orientación es paralela u oblicua al eje mayor de la FVTM.

\subsection{Falla del Bajío}

La falla del Bajío representa uno de los segmentos septentrionales del límite de la Faja Volcánica Transmexicana, ya que en el bloque del bajo se encuentra el afloramiento principal de basamento mesozoico; el volcanismo silícico oligocénico característico de la Mesa Central se encuentra localizado hacia el norte, mientras que en el bloque del alto está el volcanismo máfico miocénico-reciente característico de la FVTM. Esta falla constituye el límite norte del Bajío, región caracterizada por un bajo topográfico que va desde San Francisco del Rincón hasta la Cd. de Querétaro y donde se encuentran ubicadas las ciudades de Celaya, Salamanca, Irapuato, León, y Lagos de Moreno. Los movimientos documentados sobre esta falla a lo largo de su traza han sido de tipo normal, y su orientación es $\sim \mathrm{N} 45^{\circ} \mathrm{W}$ con inclinación hacia el suroeste, pero en su extremo oriental adquiere un rumbo $\sim \mathrm{E}-\mathrm{W}$ con echado al sur (Figura 2).

El rasgo hipsográfico de esta falla es prominente (Figura 3), el escarpe continuo y bien desarrollado mide $70 \mathrm{~km}$ de longitud. El total de desplazamiento de esta falla es poco conocido y se ha estimado únicamente en dos lugares. Uno de ellos es la región de León, Gto., donde su desplazamiento es de $850 \mathrm{~m}$, obtenido del desplazamiento de unidades litoestratigráficas oligocénicas localizadas en la parte más elevada de la sierra Cuatralba (Quintero-Legorreta, 1992) y considerando el espesor de los sedimentos dentro de la depresión del Bajío de 500 m (Hernández-Laloth, 1991), el rechazo vertical superaría los $1200 \mathrm{~m}$. El segundo lugar donde se conoce su desplazamiento es Silao, Gto. donde la falla del Bajío desplaza $500 \mathrm{~m}$ al Basalto el Cubilete fechado en 13.5 Ma por Aguirre-Díaz et al. (1997).

Esta falla ha tenido al menos tres episodios de actividad, el primero de ellos de edad eocénica fue inferido por Aranda-Gómez y McDowell (1998) a partir del basculamiento de las capas basales del Conglomerado de Guanajuato en el bloque del bajo de esta falla. El segundo evento ocurrió en el Oligoceno y fue el más importante. La falla que contiene a la Veta Madre en Guanajuato, paralela y ubicada a menos de diez kilómetros de la falla del Bajío, desplazó más de $1500 \mathrm{~m}$ a rocas de $c a$. 32 Ma y la veta que rellena la falla tiene edades de 29 a 27 Ma (Gross, 1975). Si se suma este desplazamiento al evento miocénico de la falla del Bajío en Silao, tendríamos un total que supera los 2 kilómetros en la región de Guanajuato.

\subsection{Fallas Ixtla y La Joya}

Las fallas Ixtla-La Joya forman el extremo suroriente de un pilar tectónico de rumbo NE y ca. $20 \mathrm{~km}$ de ancho, localizado entre los volcanes San Pedro y Palo Huérfano. No obstante lo tenue del rasgo morfológico actual del pilar, se infiere su importancia ya que dentro de él afloran las rocas mesozoicas; además, los volcanes poligenéticos Palo Huérfano, San Pedro, La Joya y el Zamorano, de edad Mioceno medio, están emplazados sobre su traza.

Las estructuras que constituyen las fallas Ixtla y La Joya son domos riodacíticos alineados, y fracturas y fallas normales con orientaciones que varían de $\mathrm{N} 48^{\circ} \mathrm{E}$ a $\mathrm{N} 68^{\circ} \mathrm{E}$ e inclinación hacia el sureste. Se infiere que estas fallas tuvieron un desplazamiento vertical oligocénico del orden de $250 \mathrm{~m}$ ya que las rocas mesozoicas se han encontrado en pozos a más de $350 \mathrm{~m}$ de profundidad en el graben de 
Querétaro, y las fallas de ese graben sólo tienen desplazamiento miocénico menor a $100 \mathrm{~m}$ (Alaniz-Alvarez et al., 2001). Los desplazamientos posteriores al Mioceno medio son pequeños y sólo se observan en segmentos pequeños de falla, gran parte del conjunto de fallas Ixtla-La Joya está cubierto por derrames de basalto de 6.2 y $7.5 \mathrm{Ma}$.

\subsection{Sistema de fallas La Pera}

El límite sur de la Faja Volcánica Transmexicana, en su parte central, está en gran parte oculto por depósitos volcánicos, entre los cuales se encuentran depósitos volcanicláticos de los volcanes Nevado de Toluca, Popocatépetl y del campo volcánico de la sierra de Chichinautzin. Se ha propuesto que la sierra de Chichinautzin está ubicada a lo largo de una falla con orientación E-W debido a los numerosos alineamientos de conos monogenéticos con esa orientación (e.g. Márquez et al., 1999) y al desnivel entre los afloramientos de las rocas calcáreas mesozoicas ubicadas al este de Cuernavaca a una altitud de $1840 \mathrm{msnm}$ y las reportadas en la base de los pozos de la cuenca de México, ese desnivel es de entre 1200 y $3775 \mathrm{~m}$, siendo el bloque levantado el del sur (Pérez-Cruz, 1988).

Márquez et al. (1999) presentan un estudio del alineamiento de conos monogenéticos en el campo volcánico de Chichinautzin, concluyendo que los alineamientos de más de 15 puntos tienen dos orientaciones preferenciales E-W y NE-SW. La ausencia de fallas con orientación E-W dentro de la sierra de Chichinautzin se explica porque gran parte de la extensión fue liberada por los diques que alimentaron el campo volcánico. Se ha propuesto que el material que queda bajo la superficie en magmas básicos representa de 3 a 10 veces el material que sale a la superficie (Crisp, 1984). De acuerdo con esto, en la zona de la falla de $\mathrm{La}$ Pera el emplazamiento de magmas en poco tiempo puede ser el responsable de inhibir la formación de fallas normales cuaternarias. Como es común en fallas normales, la falla mayor del sistema La Pera está sepultada, las anomalías gravimétricas reportadas en Urrutia-Fucugauchi y FloresRuiz (1996) y Ferrari et al. (2002) apoyan la existencia de esta estructura. La extensión poniente de este sistema corresponde a fallas normales con inclinación hacia el norte encontradas en Malinalco (González-Cervantes, 2004) y Tenango (Márquez et al., 1999; García-Palomo et al., 2000) .

\section{Relaciones entre fallamiento y volcanismo}

La relación entre el fallamiento y el tipo de volcanismo ha sido estudiada en la Mesa Central (Orozco-Esquivel et al., 2002), en la Faja Volcánica Transmexicana (AlanizÁlvarez et al., 1998; García-Palomo et al., 2000) y en la región de Taxco (Alaniz-Alvarez et al., 2002b, MoránZenteno et al. 2004). En la Mesa Central un campo de domos riolíticos, el cual cubre una superficie de más de
$10,000 \mathrm{~km}^{2}$, fue emplazado durante el evento oligocénico de deformación, al que se calculó $20 \%$ de alargamiento. En la Faja Volcánica Transmexicana los volcanes monogenéticos, diques y conos cineríticos están localizados a lo largo o paralelamente a las fallas normales perpendiculares a la dirección de extensión máxima (Alaniz-Alvarez et al., 1998); en la región de Taxco, en la Sierra Madre del Sur donde el sistema de fallas es lateral, se documentó el emplazamiento de domos riolíticos en un traslape extensional (Alaniz-Alvarez et al., 2002b), y, de manera similar, la caldera de Tilzapotla se emplazó en un traslape izquierdo de dos fallas laterales izquierdas (Morán-Zenteno et al., 2004); indicando en todos los casos que el emplazamiento del magmatismo ocurrió en las zonas con extensión máxima.

El hecho de que se observe que hay contemporaneidad entre el fallamiento y el volcanismo, que los magmas félsicos y básicos se hayan emplazado en zonas de extensión máxima y que el estado de esfuerzos relacionado con el emplazamiento de magmas haya sido tanto en regímenes de fallamiento lateral como normal, es consistente con lo que se ha establecido en modelos analógicos y teóricos acerca del transporte de magmas (Secor y Pollard, 1975; Takada, 1994). Se ha propuesto que para que ocurra volcanismo es necesario que haya aporte de magma, que tenga capacidad de flotación y que ascienda hacia la superficie bajo un estado de esfuerzos favorable con $\sigma_{3}$ horizontal (e.g. Takada, 1989, 1994, Watanabe et al., 1999). Las relaciones entre el volcanismo y el fallamiento pueden ser muy variadas e interdependientes. En las regiones de estudio prácticamente todos los eventos de deformación cenozoicos estudiados han ocurrido sincrónicamente con el volcanismo; pero también hemos visto que ha habido periodos de bajo aporte de magma hacia la superficie como el hiatus volcánico entre los pulsos de la Sierra Madre Occidental y de la Faja Volcánica Transmexicana y en estos casos no podemos establecer la edad máxima del fallamiento. Por ejemplo sabemos que ocurrió fallamiento sincrónico con el volcanismo oligocénico en Taxco, sin embargo no se puede saber si la deformación continuó porque no hay rocas que cubran las fallas.

\section{Edad del inicio del volcanismo en la parte central de la FVTM}

Existe un hiatus entre el volcanismo de la Sierra Madre Occidental, localizado en la Mesa Central, y el de la Faja Volcánica Transmexicana, el cual ha sido establecido para la región ubicada al oeste de la falla San Miguel de Allende (Figura 2), entre 24 y 16 Ma. La primera edad corresponde a la Ignimbrita San Nicolás, la cual es la ignimbrita riolítica más joven reconocida en el límite sur de la Mesa Central (Nieto-Samaniego et al., 1996; Ojeda-García, 2004) y la segunda corresponde a las rocas volcánicas que subyacen a los derrames del volcán Palo Huérfano, el cual se considera que forma parte de los primeros volcanes poligenéticos de 
la FVTM (Pérez-Venzor et al., 1996). Edades similares para este hiatus han sido establecidos por Cerca-Martínez et al. (2000) entre 22 y 14.6 Ma en la sierra de Guanajuato, y entre 22 y 10 Ma por Verma y Carrasco-Núñez (2003) para la región del volcán El Zamorano (100¹0'-2055').

El volcanismo más joven en la región de Taxco, que corresponde a la parte central-septentrional de la provincia magmática del sur de México, es de 31.6 Ma (AlanizAlvarez et al. 2002b) y corresponde a domos y flujos piroclásticos, ambos de composición riolítica. En la región de Toluca y Malinalco, el volcanismo andesítico más viejo corresponde a la Andesita basáltica San Antonio de 21.6 Ma (García-Palomo et al., 2000). El volcanismo basáltico ha sido reportado en el límite sur de la FVTM con edades entre 20.5 y 16.7 Ma (Ferrari et al., 2003b). En la región Taxco-Tepoztlán, el hiatus volcánico entre el volcanismo riolítico, predominante en la Sierra Madre Occidental, y el basáltico de la FVTM estaría entre los 31 y $21 \mathrm{Ma}$.

Tanto al norte y sur de la FVTM como al este y oeste del SFTSMA, se registra un hiatus volcánico en varias localidades, que duró entre 6 y $12 \mathrm{Ma}$. Hay que aclarar que hubo volcanismo entre los 30 y $16 \mathrm{Ma}$ en otras localidades dentro de la región considerada en este estudio (Ferrari et al., 1999, García-Palomo et al., 2002; Cerca-Martínez, 2004) pero su importancia volumétrica relativa es mucho menor. Lo anterior parece indicar que el evento geológico que dio origen a la Faja Volcánica Transmexicana estuvo separado en el tiempo de aquellos que dieron origen a las provincias volcánicas de la Sierra Madre Occidental y de la Sierra Madre del Sur.

Las rocas cenozoicas más antiguas reportadas en pozos perforados en la Ciudad de México corresponden a basaltos y tobas oligocénicas. Con base en sísmica de reflexión e información obtenida de pozos en la Ciudad de México, Pérez-Cruz (1988) documentó que la base de la cuenca de México es irregular y que el basamento mesozoico está a profundidades de $1600 \mathrm{~m}$ en el pozo Mixhuca-1 y más de 3200 m en el pozo Roma-1 (Figura 4). También se han reportado 5 fechamientos que van de 31.4 Ma a 23 Ma en muestras obtenidas entre 1000 y 2000 m de profundidad (Marsal y Graue, 1969; Oviedo, 1970; Pérez-Cruz, 1988).

\section{Discusión}

En este trabajo se ha tratado de establecer las condiciones bajo las cuales la FVTM se ha convertido en una frontera tectónica, es decir el límite que divide terrenos con distinta historia geológica o estructural a partir de cierto tiempo. Se puede hablar de frontera tectónica a distintas escalas; considerando que ésta representa una región que acomoda la deformación entre dominios estructurales distintos, esta región puede ser tan amplia como una provincia geológica (Faja Volcánica Transmexicana), o bien puede estar formada por un sistemas de fallas (sistema de fallas
Taxco-San Miguel de Allende), o ser tan discreta como una falla mayor (e.g. falla del Bajío). Las fronteras separan regiones con historias geológicas diferentes en distintas escalas de tiempo y espacio, y han tenido una influencia importante en el registro estratigráfico de las regiones donde se localizan.

La FVTM se estableció como un arco volcánico a partir del Mioceno medio, aunque su historia como frontera tectónica inició mucho antes. De acuerdo con estudios previos sobre la parte central de México discutidos en este trabajo, durante el Cretácico no hubo una diferencia notable entre los bloques ubicados al sur y norte de la FVTM, ni en el tipo de litología ni en su estructura. Existió un ambiente marino de plataforma hacia el oriente con arcos volcánicos localizados hacia el extremo poniente y la orogenia Laramide estuvo presente prácticamente con el mismo estilo y edad de deformación al norte y sur de la FVTM.

Los datos y observaciones presentados en este trabajo no permiten establecer diferencias notables para el Paleoceno. La presencia de estructuras contractivas con vergencia hacia el occidente se documentó únicamente en la parte septentrional-central de la Sierra Madre del Sur. Esta deformación ocurrió a fines del Cretácico y principios del Cenozoico y no está presente en todos los sitios. En Tierra Caliente, Salinas-Prieto et al., (2000) documentaron que esta deformación fue de baja intensidad, mientras que en el borde oriente de la plataforma Guerrero-Morelos fue más intensa (Cerca-Martínez, 2004). Esta variación en el sentido de vergencia de las estructuras contractivas, que no ha sido documentada al norte de la FVTM, no se considera suficientemente importante como para considerar que la FVTM actuó como una frontera tectónica durante ese evento de deformación.

Durante el Eoceno medio y tardío las deformaciones ocurridas al norte y sur de la Faja Volcánica Transmexicana muestran diferencias notables. En la Sierra de Guanajuato se documentó un evento de extensión postlarámide liberado por fallas normales cuya edad de actividad cubre gran parte del Eoceno (Aranda-Gómez y McDowell, 1998). En Taxco, al sur de la Faja Volcánica Transmexicana, no se pudo establecer el origen de los depósitos de conglomerados rojos postlaramídicos. Para fines del Eoceno (entre 36 y $33 \mathrm{Ma}$ ), se documentó una fase de fallamiento lateral que generó alargamiento horizontal hacia el NNW. Esta fase se caracteriza por el desplazamiento lateral izquierdo de fallas paralelas a la costa y se encuentra documentada en Taxco (Alaniz-Alvarez et al., 2002b), Tierra Colorada (Riller et al., 1992), Tilzapotla (Morán-Zenteno et al., 20004) y Huatulco (Tolson, 1998), siendo más joven la deformación hacia el oriente. Éste es el tiempo en el que se marca la primera separación entre los dominios estructurales ubicados en los extremos norte y sur de la Faja Volcánica Transmexicana.

Durante el Oligoceno continuó la separación entre la deformación ocurrida en la Mesa Central y en la Sierra Madre del Sur, mientras que al norte de la Faja Volcánica 

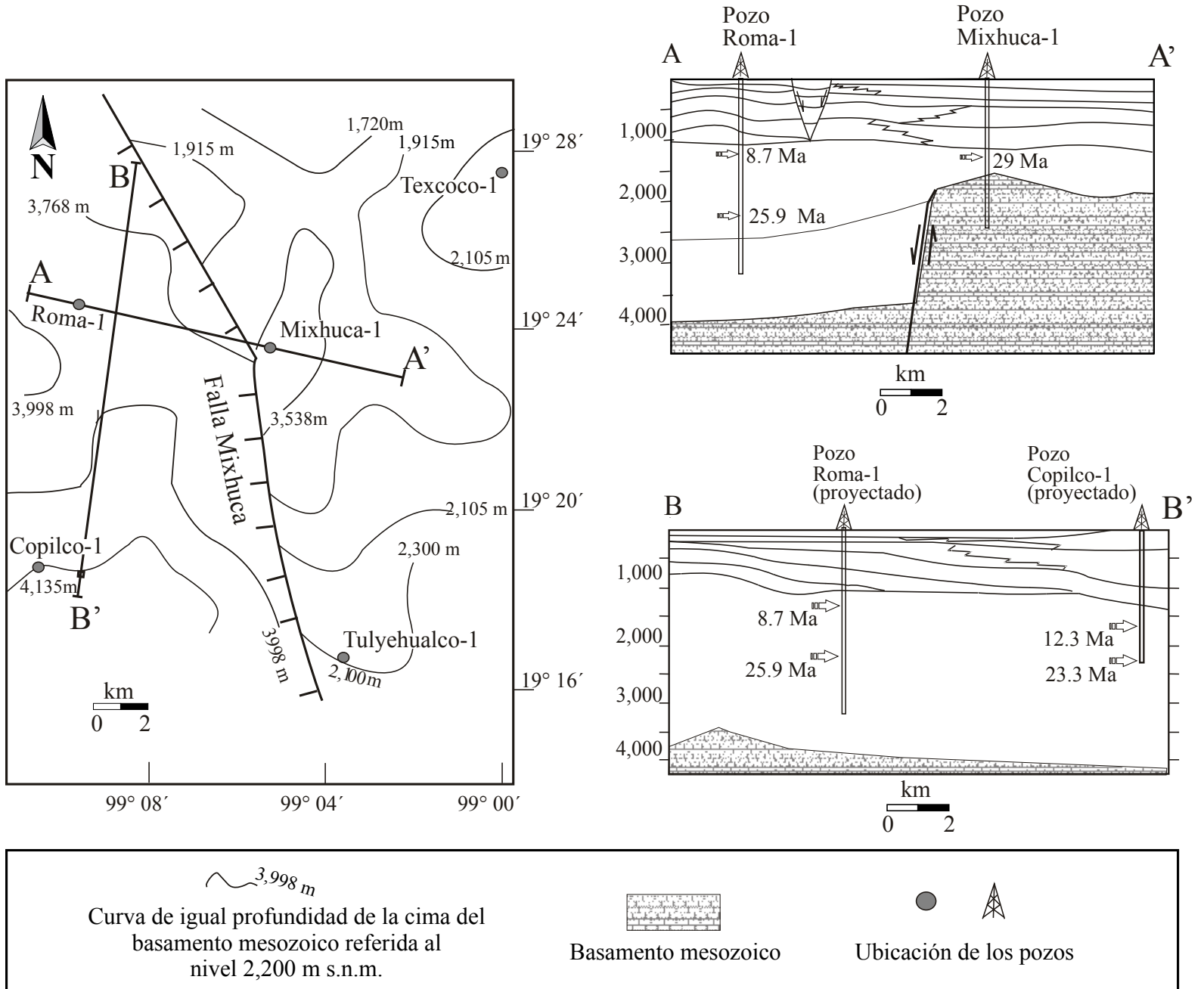

Basamento mesozoico

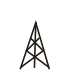

Ubicación de los pozos

Figura 4. a) Configuración de las líneas de igual profundidad de la cima del basamento mesozoico en la Cuenca de México, obtenidas con datos de pozos y líneas sísmicas de reflexión. b) Secciones geológicas. Tomado de Pérez-Cruz (1988).

Transmexicana ocurrió deformación extensional, al sur ocurrió deformación transcurrente. En la Mesa Central, Nieto-Samaniego et al., (1999) documentaron que la mayor extensión fue de $c a$. 20\% hacia el ENE-WSW, también ocurrió extensión horizontal en la dirección NNW-SSE de $11 \%$ y acortamiento en la vertical. Esa deformación migró hacia el oeste y sureste, esos autores proponen que dicha migración fue controlada por cambios en la velocidad de acreción en la placa Pacífico y el ángulo de subducción de ésta por debajo de la placa Norteamérica.

En contraste, en la región de Taxco se documentó que la extensión horizontal generada por la tectónica trascurrente oligocénica fue hacia el NE-SW y el acortamiento hacia el NW-SE. Este evento migró hacia el oriente y se registró principalmente por fallamiento normal en la fosa formada por la falla de Oaxaca, cuya edad varía de anterior al Oligoceno en las cercanías de Tehuacán a Mioceno temprano en la región de Etla y la ciudad de Oaxaca (NietoSamaniego et al., en prensa).
Desde el Mioceno medio se ha concentrado la deformación extensional a lo largo de la Faja Volcánica Transmexicana contemporáneamente con un gran pulso magmático. La deformación ha sido liberada principalmente por fallas normales con rumbos ENE-WSW en el sistema Chapala-Tula, aunque también se han activado fallas normales con rumbo NNW-SSE del sistema de fallas Taxco-San Miguel de Allende y NE-SW en la región de Apan, Hidalgo.

De los eventos analizados se infiere que a partir del Eoceno tardío se generó una discontinuidad cortical en el sitio ocupado por la parte central de la Faja Volcánica Transmexicana (Figura 3). Esta idea surge al considerar que durante la deformación transcurrente ocurrida en la Sierra Madre del Sur durante el Eoceno tardío y OligocenoMioceno, en la plataforma Valles-San Luis Potosí no hubo deformación importante y que la migración de las deformaciones fue en sentidos contrarios, al norte de la FVTM migró hacia el oeste y al sur de la FVTM migró hacia el este. 
Estas circunstancias hacen necesario considerar que entre las regiones localizadas al norte y sur de la FVTM debió existir una zona que permita acomodar esa incompatibilidad en la deformación. Las evidencias disponibles expuestas en párrafos anteriores indican que en la cuenca de México la zona de acomodo formó un grupo de fallas actualmente sepultadas por las rocas volcánicas del Mioceno-Holoceno. Parece razonable suponer también la presencia de una zona de acomodo en la parte más occidental de la FVTM ya que Rosas-Elguera et al. (1996) y Ferrari y Rosas-Elguera (2000) consideran que en esa región la FVTM reposa sobre un límite cortical mayor entre el Bloque Jalisco y la Sierra Madre Occidental individualizado desde el Eoceno.

\subsection{Deformación en las etapas iniciales de la FVTM}

La deformación ocurrida en las etapas iniciales de la formación de la FVTM es difícil de establecer, ya que la mayoría de las estructuras producidas en aquel tiempo están cubiertas por una pila volcánica de hasta $4 \mathrm{~km}$. Afortunadamente para la parte central-oriental de la FVTM se cuenta con los datos del subsuelo de la cuenca de México (Marsal y Graue, 1969; Oviedo, 1970; Pérez-Cruz, 1988). El hecho de que la cuenca alcance más de $4000 \mathrm{~m}$ de profundidad y que esté afectada por fallas nos indica que su formación estuvo relacionada con uno o varios eventos de deformación.

El desnivel entre el afloramiento de las rocas calcáreas marinas en Cuernavaca y en el subsuelo de la cuenca de México es el punto de partida para reconstruir la evolución de la cuenca. En el subsuelo, la configuración del contacto entre las rocas mesozoicas y el relleno de la cuenca indica la existencia de una falla normal de dirección NNW-SSE, con buzamiento al poniente, denominada falla Mixhuca. La falla presenta un rechazo vertical de entre 1600 y 2000 $\mathrm{m}$ medido por el espesor del relleno y está sepultada por las rocas de edad miocénica. Esa estructura fue interpretada a partir de los perfiles sísmicos y cortes litológicos en los pozos Roma-1, Mixhuca-1, Copilco-1, Tulyehualco-1 (Pérez-Cruz, 1988) y Texcoco (Oviedo, 1970) (Figura 4).

El registro litológico de los pozos muestra con claridad que las rocas más antiguas del relleno de la cuenca son anteriores al Oligoceno tardío. En el pozo Texcoco, la columna estratigráfica reportada por Oviedo (1970) muestra una secuencia volcaniclástica que va de los 480 a $1965 \mathrm{~m}$ de profundidad, hacia los $1500 \mathrm{~m}$ reporta edades de $31.4 \mathrm{y}$ 30 Ma obtenidas por Marsal y Graue (1969); debajo de esa secuencia aparecen $c a .100 \mathrm{~m}$ de anhidritas continentales, las cuales a su vez yacen sobre el conglomerado Texcoco, descrito como un conglomerado continental cuya edad es desconocida, pero que contiene cantos de calizas del Cretácico Superior; el espesor del conglomerado puede estimarse considerando la profundidad del basamento reportada por Pérez-Cruz (1988), que para la zona de Texcoco es un poco mayor que $2105 \mathrm{~m}$, lo que indicaría un espesor del conglomerado cercano a $50 \mathrm{~m}$. En el pozo Roma-1 la edad isotópica más antigua que se tiene es de 25.9 Ma y se localiza a los $2200 \mathrm{~m}$ de profundidad, debajo yacen alrededor de $\sim 1700 \mathrm{~m}$ de relleno, medidos hasta alcanzar la cima de las rocas mesozoicas. En el pozo Copilco-1 la edad más antigua es de 23.3 Ma y se localiza a $2250 \mathrm{~m}$ de profundidad, debajo hay $\sim 1900 \mathrm{~m}$ de relleno. En el pozo Mixhuca las rocas volcánicas más antiguas son de $29 \mathrm{Ma}$ y se localiza a $1200 \mathrm{~m}$ de profundidad, debajo hay $c a .220$ $\mathrm{m}$ de relleno. La diferencia de espesores del relleno de la cuenca entre los pozos Texcoco, Mixhuca-1 y Tulyehualco1, localizados en el bloque levantado de la falla Mixhuca, y los de los pozos Roma-1 y Copilco localizados en el bloque hundido muestran que este último tiene un relleno alrededor de dos kilómetros más grueso; además indican que la actividad de dicha estructura fue anterior al Mioceno temprano ya que sedimentos de esa edad sepultan a la falla.

La edad de los rellenos más profundos se desconoce, sin embargo, como una aproximación (se omiten correcciones por compactación) podemos considerar que en el pozo Roma-1 los 2200 m superiores de relleno se depositaron a una tasa cercana a $80 \mathrm{~m}$ por millón de años y en el pozo Copilco-1 a una tasa de 98 m por millón de años. Si consideramos tasas de sedimentación similares para el relleno más profundo (por debajo de los $2200 \mathrm{~m}$ hasta $4100 \mathrm{~m}$ ), podríamos suponer que su depósito abarca un lapso de entre 15 y $20 \mathrm{Ma}$. Siendo conservadores, nosotros suponemos que ese depósito debió demorar al menos $10 \mathrm{Ma}$, lo que nos indicaría que la edad de inicio del relleno de la cuenca es del Eoceno tardío. Esta estimación es consistente con las edades obtenidas en los pozos Texcoco y Mixhuca-1 donde se reportaron las rocas del Oligoceno temprano y bajo las cuales los espesores del relleno de la cuenca son un poco mayores a los $200 \mathrm{~m}$. Debe considerarse además, que el registro estratigráfico del pozo Texcoco muestra para los $150 \mathrm{~m}$ inferiores, anhidrita y conglomerado, cuyas tasas de sedimentación probablemente fueron menores que las del relleno volcaniclástico.

Otra observación importante de la configuración de la cima de las rocas mesozoicas en la cuenca de México es que, tanto en el bajo como en el alto de la falla Mixhuca, esa superficie muestra una pendiente con inclinación hacia el sur (Figura 4, Pérez-Cruz, 1988, figura 15). Los cortes de la cima de las rocas mesozoicas en los pozos Roma-1 y Copilco-1 localizados en el alto de la falla Mixhuca muestran un desnivel de $210 \mathrm{~m}$ inclinándose hacia el sur. Los cortes de la cima de las rocas mesozoicas en los pozos Mixhuca-1 y Tulyehualco-1 localizados en el bajo de la falla Mixhuca muestran un desnivel de $525 \mathrm{~m}$ también inclinándose hacia el sur (Figura 4).

La distribución de las rocas mesozoicas y los desniveles entre sus afloramientos al sur de la FVTM en Cuernavaca, y en el subsuelo de la cuenca de México indican la presencia de fallas orientadas este-oeste, localizadas hacia el límite sur de dicha cuenca. Ese sistema de fallas ha sido propuesto por algunos autores denominándolo sistema de fallas La 
Pera y ubicándolo bajo la sierra de Chichinautzin (Ferrari et al. 2002; Siebe et al., 2004; Delgado-Granados et al., 1995). La configuración sísmico-estructural reportada por Pérez-Cruz (1988, figura 16) muestra para el límite entre las unidades "volcánica inferior" y "volcánica superior", cuya edad se localiza a finales del Mioceno medio según las edades reportadas en ese trabajo, un patrón con múltiples escarpes (zonas de mayor pendiente) orientados este-oeste, observándose también que en gran parte de la cuenca la secuencia miocénica tiene una pendiente general que se inclina hacia el sur. Estos datos son compatibles con la existencia de una gran falla maestra localizada al sur de la cuenca, cuya actividad fue posterior al desplazamiento de $1600 \mathrm{~m}$ de la falla Mixhuca, dado que bascula de manera semejante al bajo y al alto de esa estructura.

De la información expuesta arriba inferimos la siguiente secuencia de eventos en la cuenca de México: durante el Paleoceno temprano, quizá incluyendo la parte superior del Maastrichtiano de acuerdo con las edades de Formación Mexcala en Texmalac, ocurrió la deformación laramídica que plegó los sedimentos marinos mesozoicos. Posteriormente tuvo lugar un estadío de depósito de molasas continentales representadas por el conglomerado Texcoco de cantos de caliza sin contenido volcánico, observado en los pozos de la cuenca de México, al cual siguió un estadío de menor actividad tectónica y nula o mínima actividad volcánica, durante el que se depositaron las anhidritas. El inicio intempestivo de volcanismo y la actividad tectónica en la falla Mixhuca ocurrió antes del Oligoceno temprano acumulándose en el bloque hundido de la falla Mixhuca más de dos mil metros de material volcaniclástico principalmente. La actividad volcánica se mantuvo hasta el Reciente y la actividad tectónica se centró en la falla $\mathrm{La}$ Pera a partir del Oligoceno.

De acuerdo con lo anterior, tuvieron lugar dos eventos principales de deformación, uno de edad Eoceno tardío durante el cual la deformación fue acomodada principalmente por la falla Mixhuca con alargamiento horizontal orientado E-W o WSW y el otro evento ocurrido durante el Oligoceno-Mioceno y su deformación fue acomodada principalmente en la falla La Pera, con alargamiento N-S o NNW.

Si comparamos los eventos de deformación descritos en los párrafos de arriba con aquellos documentados al norte y sur de la FVTM (Figura 3C), vemos que para el Eoceno - Mioceno, la deformación fue mínima o nula en la Sierra Madre Oriental; en todo caso la poca deformación sería con fallamiento normal y dirección de extensión $\sim \mathrm{E}-\mathrm{W}$; mientras que en la Sierra Madre del Sur hubo dos fases de deformación, en ambos casos de tipo transcurrente, una de edad Eoceno - inicio del Oligoceno con dirección de alargamiento horizontal $\sim$ NW-SE y acortamiento $\sim$ NE-SW, y la segunda de edad Oligoceno-Mioceno con dirección de alargamiento $\sim$ NE-SW y dirección de acortamiento NWSE. Considerando toda la región estudiada, es claro que se trata en ambos casos de una deformación heterogénea
(Figura 3C) y que el alargamiento máximo resultante en la zona de cizalla simple localizada entre los dos dominios estructurales (zona de falla La Pera) dependerá de las magnitudes de la vorticidad y la extensión impuestas en la región localizada al sur de la zona de cizalla. Para el Oligoceno-Mioceno la dirección de máximo alargamiento predicha dentro de la FVTM es en el cuadrante NW-SE sin que se pueda determinar una orientación precisa, a menor cantidad de rotación la dirección de máximo alargamiento se aproximará a la dirección N-S lo que produciría desplazamiento vertical en el sistema de fallas La Pera (Figura 3C). En el caso de la deformación del Eoceno tardío, cuando tuvo lugar el desplazamiento normal en la falla Mixhuca, se infiere que a lo largo de la zona de falla La Pera el deslizamiento tuvo una componente dominante lateral derecha debido a la extensión E-W documentada dentro de la cuenca, las magnitudes del desplazamiento dependerán principalmente de la magnitud de esa extensión.

A partir de la información con que actualmente se cuenta y aunque aún se encuentra documentado en pocos lugares, la FVTM se aprecia como una gran zona de acomodo entre deformaciones incompatibles ocurridas en los bloques corticales localizados al norte y sur de ella.

\section{Conclusiones}

El estudio de las deformaciones ocurridas en la Mesa Central, Sierra Madre Oriental y Sierra Madre del sur en el centro de México nos permiten concluir que:

- El sistema de fallas Taxco San Miguel de Allende se ubica en el sitio de transición entre una zona de plataformas continentales y una de mayor batimetría. Esta discontinuidad paleogeográfica de edad cretácica controló la localización de fallamiento cenozoico de rumbo N-S y NNW-SSE.

- Desde el Eoceno la deformación extensional de la Mesa Central y transcurrente de la Sierra Madre del Sur indica que existió una zona de despegue entre estas provincias, en el sitio ocupado actualmente por la Faja Volcánica Transmexicana.

- En el Oligoceno la actividad de las fallas laterales en el sur de México implicó el acortamiento en la dirección NW-SE y extensión al NE-SW; en la Mesa Central ocurrió deformación extensional en dos direcciones $\sim \mathrm{E}-\mathrm{W}$ y $\sim \mathrm{N}$ S y en la Sierra Madre Oriental, al norte de la FVTM, no hubo deformación cenozoica significativa.

- Del Mioceno al Reciente la deformación se ha concentrado en la Faja Volcánica Transmexicana.

- La correlación de los datos obtenidos en los pozos y estudios de reflexión sísmica, reportados por Pérez-Cruz (1988) para la cuenca de México, así como los eventos de deformación analizados en este trabajo, indican que desde el Eoceno tardío la deformación en la parte meridional-central de la FVTM fue una respuesta de las deformaciones en los bloques que la limitan al norte y sur. Para la cuenca de 
México, en el Eoceno tardío la actividad mayor se localizó sobre la falla de Mixhuca ocurriendo alargamiento horizontal orientado E-W o WSW. Durante el Oligoceno-Mioceno la deformación fue acomodada principalmente en la falla La Pera, con alargamiento N-S o NNW.

\section{Agradecimientos}

Los autores agradecen las revisiones de Dante J. Morán Zenteno, Álvaro Márquez y Luca Ferrari, que permitieron mejorar el artículo. El financiamiento de esta investigación fue proporcionado por el Consejo Nacional de Ciencia y Tecnología con el proyecto 41044-F y por la Universidad Nacional Autónoma de México con el proyecto PAPIITIN102602.

\section{Referencias bibliográficas}

Aguilera-Franco, N., 2003, Cenomanian-Coniacian zonation (foraminifers and calcareous algae) in the Guerrero - Morelos basin, southern México: Revista Mexicana de Ciencias Geológicas, 20, 202-222.

Aguirre-Díaz, G. J., Nelson, S. A., Ferrari, L., López-Martínez, M., 1997, Ignimbrites of the central Mexican Volcanic Belt, Amealco and Huichapan Calderas (Querétaro-Hidalgo), en International Association of Volcanology and Chemistry of the Earth Interior General Assembly 1997, Field Trip \#1 Guidebook: Puerto Vallarta, México, $54 \mathrm{p}$.

Alaniz-Alvarez, S.A., Nieto-Samaniego, A. F., Ferrari, L., 1998, Effect of the strain rate in the distribution of monogenetic and polygenetic volcanism in the Transmexican Volcanic Belt: Geology, 26, 591-594.

Alaniz-Alvarez, S.A., Nieto-Samaniego, A.F., Reyes-Zaragoza, M.A., Orozco-Esquivel, M.T., Ojeda-García, A.C., Vasallo-Morales, L. F., 2001, Estratigrafía y deformación de la región San Miguel de Allende-Querétaro: Revista Mexicana de Ciencias Geológicas, $18,129-148$.

Alaniz-Alvarez, S.A., Nieto-Samaniego, A.F., Orozco-Esquivel, M.T., Vasallo-Morales, L. F., Xu, S.S., 2002a, El Sistema de Fallas TaxcoSan Miguel de Allende: Implicaciones en la deformación postEocénica del centro de México: Boletín de la Sociedad Geológica Mexicana, 55, 12-29.

Alaniz-Alvarez, S. A., Nieto-Samaniego, A. F., Morán-Zenteno, D. J., Alba-Aldave, L., 2002b, Rhyolitic volcanism in extension zone associated with strike-slip tectonics in the Taxco region, Southern México: Journal of Volcanology and Geothermal Research, 118, $1-14$.

Alencaster, G., 1980, Moluscos del Maastrichtiano de Texmalac, Guerrero: Sociedad Geológica Mexicana, Libro guía de la excursión geológica a la cuenca del alto Río Balsas, 39-42.

Anderson, T.H. Silver, L.T., 1974, Late Cretaceous plutonism in Sonora, Mexico, and its relationship to circum-Pacific magmatism: Geological Society of America Rocky Mountain Section Annual Meeting Program, 484.

Aranda-Gómez, J.J., McDowell, F.W., 1998, Paleogene extension in the southern Basin and Range Province of Mexico: Syn-depositional tilting of Eocene red beds and Oligocene volcanic rocks in the Guanajuato mining district: International Geology Review, 40, 116-134.

Aranda-Gómez, J.J., Henry, C.D., Luhr, J.F., 2000, Evolución tectonomagmática post-paleocénica de la Sierra Madre Occidental y de la porción meridional de la provincia tectónica de Cuencas y Sierras, México: Boletín de la Sociedad Geológica Mexicana, $53,59-71$.
Cabral-Cano, E., Draper, G., Lang, H.R., Harrison, C.G.A., 2000, Constraining the late Mesozoic and early Tertiary tectonic evolution of southern Mexico: structure and deformation history of the Tierra Caliente region, southern Mexico: Journal of Geology, 108, 427-446.

Campa, M.F., Coney, P.J., 1983, Tectonostratigraphic terranes and mineral resource distribution in Mexico: Canadian Journal of Earth Sciences, 20, 1040-1051.

Carrillo Bravo, J., 1971, La Plataforma de Valles-San Luis Potosí: Boletín de la Asociación Mexicana de Geólogos Petroleros, 23, 1-110.

Centeno-García, E., Silva-Romo, G., 1997, Petrogenesis and tectonic evolution of central Mexico during Triassic-Jurassic time: Revista Mexicana de Ciencias Geológicas, 14, 244-260.

Cerca-Martínez, M., 2004, Deformación y magmatismo Cretácico TardíoTerciario temprano en la zona de la Plataforma Guerrero-Morelos, México D.F., Universidad Nacional Autónoma de México, Tesis doctoral, $17 \mathrm{p}$.

Cerca-Martínez, L. M., Aguirre-Díaz, G. López-Martínez M., 2000, The geologic evolution of the southern sierra de Guanajuato, México: A Documented Example of the Transition from the Sierra Madre Occidental to the Mexican Volcanic Belt: International Geology Review, 42, 131-151.

Cerca, M., Ferrari, L., Bonini, M., Corti, G., Manetti, P., 2004, The role of crustal heterogeneity in controlling vertical coupling during Laramide shortening and the development of the Caribbean-North America transform boundary in southern Mexico: insights from analogue models, en Grocott, J., Taylor G., Tikoff, B. (eds.), Vertical coupling and decoupling in the Lithosphere: Geological Society, Special Publication 227, 117-140.

Crisp, J. A., 1984, Rates of magma emplacement and volcanic output: Journal of Volcanology and Geothermal Research, 20, 177-211.

Dávalos-Álvarez, O., 2004, Evolución de las fallas mayores del NeógenoCuaternario en la región de Huimilpan, Querétaro, San Luis Potosí, México, Universidad Autónoma de San Luis Potosí, Facultad de Ingeniería, Tesis de licenciatura, $93 \mathrm{p}$.

De Cerna, Z., Ortega-Gutiérrez, F., Palacios-Nieto, M., 1980, Reconocimiento geológico de la parte central de la cuenca del alto Río Balsas, Estados de Guerrero y Puebla: Sociedad Geológica Mexicana, Libro guía de la excursión geológica a la cuenca del alto Río Balsas, 1-33.

Delgado-Granados, H., Nieto-Obregón, J., Silva-Romo, G., MendozaRosales, C.C., Arellano-Gil, J., Lermo-Samaniego, J.F., RodríguezGonzález, M., 1995, La Pera detachment fault system: active faulting south of México City (II): geological evidence: GEOS, 15,64 .

Demant, A., 1978, Características del Eje Neovolcánico Transmexicano y sus problemas de interpretación: Universidad Nacional Autónoma de México, Instituto de Geología, Revista, 2, 172-187.

Dubois, A.., Odonneb, F., Massonnatc, G., Lebourgd, T., Fabred, R., 2002, Analogue modelling of fault reactivation: tectonic inversion and oblique remobilisation of grabens: Journal of Structural Geology, 24, 1741-1752.

Edwards, J. D., 1955, Studies of some Early Tertiary red conglomerates of Central Mexico: U.S. Geological Survey Professional Paper 264-H, $183 \mathrm{p}$.

Eguiluz de Antuñano, S., Aranda-García, M., Marrett, R., 2000, Tectónica de la Sierra Madre Oriental: Boletín de la Sociedad Geológica Mexicana, 53, 1-26.

Ferrari, L., Rosas-Elguera, J., 2000, Late Miocene to Quaternary extension at the northern boundary of the Jalisco block, western Mexico: the Tepic-Zacoalco rift revised: Geological Society of America Special Paper, 334, 42-64.

Ferrari, L., López-Martínez, M., Aguirre-Díaz, G., Carrasco-Nuñez, G., 1999, Space-time patterns of Cenozoic arc volcanism in central Mexico: from the Sierra Madre Occidental to the Mexican Volcanic Belt: Geology, 27, 303-306.

Ferrari, L., Mena, M., López-Martínez, M., Jacobo-Albarrán, J., SilvaRomo, G., Mendoza-Rosales, C. C., González-Cervantes, N., 2002, Estratigrafía y Tectónica de la cuenca de la Ciudad de México y áreas colindantes, Geos, 22, 150. 
Ferrari, L., Petrone, C. M., Francalanci, L., Tagami, T., Eguchi, M., Conticelli, S., Manetti, P., Venegas-Salgado S., 2003a, Geology of the San Pedro-Ceboruco graben, western Trans-Mexican Volcanic Belt: Revista Mexicana de Ciencias Geológicas, 20, 165-181.

Ferrari, L., López-Martínez, M., González-Cervantes, N., JacoboAlbarrán, J., Hernández-Bernal, M. S., 2003b, Volcanic record and age of formation of the Mexico city basin: GEOS, 23, 120.

Ferrari, L., Valencia-Moreno, M., Bryan, S., 2005, Magmatismo y tectónica en la Sierra Madre Occidental y su relación con la evolución de la margen occidental de Norteamérica, Boletín de la Sociedad Geológica Mexicana, 57(3).

Flores, T., 1920, Estudio Geológico Minero de los distritos de El Oro y Tlalpuhahua, Boletín del Instituto de Geología, 37, 40 p.

Freydier, C., Lapierre, H., Ruíz, J., Tardy, M., Martínez-R,J. Coulon, C., 2000, The Early Cretaceous Arperos basin: an oceanic domain dividing the Guerrero arc from nuclear Mexico evidenced by geochemistry of the lavas and sediments: Journal of South America Earth Sciences, 13, 325-336.

García-Palomo, A., Macías, J. L. Garduño, V. H., 2000, Miocene to Recent structural evolution of the Nevado de Toluca volcano region, central México: Tectonophysics, 318, 281-302.

García-Palomo, A., Macías, J. L., Tolson, G., Valdéz, G., Mora, J. C., 2002, Volcanic Stratigraphy and geological evolution of the Apan region, east-central sector of the Trans-Mexican Volcanic Belt: Geofísica Internacional, 41, 133-150.

González-Cervantes, N., 2004, Influencia de la deformación extensional en el vulcanismo al sur de la Cuenca de México: Cd. Madero, Tamps., Instituto Tecnológico de Ciudad Madero, Tesis de licenciatura, 108 p., 1 mapa anexo.

Gross, W. H., 1975, New ore discovery and source of silver-gold veins, Guanajuato, Mexico: Economic Geology, 70, 1175-1189.

Hernández-Jáuregui, R., Valencia-Islas, J. J., González-Casildo, V., 2000, Facies turbidíticas relacionadas a movimientos orogénicos en el centro oriente del Estado de Querétaro, México: Geos, 20, 156.

Hernández-Laloth, N., 1991, Modelo conceptual de funcionamiento hidrodinámico del sistema acuífero del valle de León, Guanajuato: México, D. F., Universidad Nacional Autónoma de México, Facultad de Ingeniería, Tesis de licenciatura, 129 p.

Hernández-Romano, U., Aguilera-Franco, N., Martínez-Medrano, M., Barceló-Duarte, J., 1997, Guerrero-Morelos Platform drowning at the Cenomanian - Turonian boundary, Huitziltepec area, Guerrero State, southern Mexico: Cretaceous Research, 18, 661-686.

Imlay, R.W., 1938, Studies of the Mexican Geosyncline: Bulletin of the Geological Society of America, 49, 1661-1694.

Marquez, A., Verma, S. P., Anguita, F., Oyarzun, R., Brandle, J. L., 1999, Tectonics and volcanism of Sierra Chichinautzin: extension at the front of the Central Trans-Mexican Volcanic belt: Journal of Volcanology and Geothermal Research, 93, 125-150.

Marsal, R. J., Graue, R., 1969, El subsuelo del lago de Texcoco, en Carrillo Nabor, El hundimiento de la Ciudad de México y Proyecto Texcoco, México, D. F., Secretaría de Hacienda y Crédito Público, $167-202$

Martínez-Reyes, J., 1992, Mapa geológico de la sierra de Guanajuato, escala 1:100,000, con Resumen de la geología de la sierra de Guanajuato México, D. F., Instituto de Geología, Universidad Nacional Autónoma de México.

Martínez-Reyes, J., Nieto-Samaniego, A. F., 1990, Efectos geológicos de la tectónica reciente en la parte central de México: Universidad Nacional Autónoma de México, Instituto de Geología, Revista, 9, 33-50.

Mooser, F., 1972, The Mexican Volcanic Belt: structure and tectonics: Geofísica Interacional, 12, 55-70.

Morán-Zenteno, D. J., Urrutia-Fucugauchi, J., Böhnel, H., GonzálezTorres, E., 1988, Paleomagnetismo de rocas jurásicas del norte de Oaxaca y sus implicaciones tectónicas: Geofísica Internacional, $27,485-518$

Morán-Zenteno, D. J. , Martiny, B., Tolson, G. , Solís-Pichardo, G., AlbaAldave, L., Hernández-Bernal, M. S., Macías-Romo, C., MartínezSerrano, R. G., Schaaf, P., Silva Romo, G., 2000, Geocronología y características geoquímicas de las rocas magmáticas terciarias de la Sierra Madre del Sur: Boletín de la Sociedad Geológica Mexicana, 53, 27-58.

Morán-Zenteno, D. J., Alba-Aldave, L. A., Solé, J., Iriondo, A., 2004 A major resurgent caldera in southern Mexico: the source of the late Eocene Tilzapotla ignimbrite: Journal of Volcanology and Geothermal Research, 136, 97-119.

Moreira-Rivera, F., Martínez-Rodríguez, L., Palacios-García, R., Maldonado-Lee, J. M., Olvera-Campos, A., Mata-Pérez, F., PérezBenavides, A., González-Monsivais, P., 1996, Carta geológico-minera Matehuala F14-1, escala 1:250,000: Pachuca, Hidalgo, México, Consejo de Recursos Minerales, 1 mapa.

Nieto-Samaniego, A. F., Alaniz-Alvarez, S. A., 1994, La Falla de San Miguel de Allende: características y evidencias de su actividad cenozoica (resumen), en Tercera Reunión Nacional de Geomorfología: Guadalajara, Jal., México, Sociedad Mexicana de Geomorfología, 139-142.

Nieto-Samaniego, A. F., Macías-Romo C., Alaniz-Alvarez, S. A., 1996, Nuevas edades isotópicas de la cubierta volcánica cenozoica de la parte meridional de la Mesa Central, México: Revista Mexicana de Ciencias Geológicas, 13, 117-122.

Nieto-Samaniego, A. F., Ferrari, L., Alaniz-Alvarez, S. A., LabartheHernández, G., Rosas-Elguera, J., 1999, Variation of Cenozoic extension and volcanism across the southern Sierra Madre Occidental Volcanic Province, México: Geological Society of America Bulletin, 111, 347-363.

Nieto-Samaniego A. F., Alaniz-Alvarez, S. A., Silva-Romo, G., EguizaCastro, M. H., Mendoza-Rosales, C. C., en prensa, Maastrichtian to Miocene main deformation events in eastern Sierra Madre del Sur, Mexico, inferred from the geometry and age of major structures, Geological Society of America Bulletin.

Ojeda-García, A. C., 2004, Cartografía geológica 1:50,000 del límite El Bajío-Mesa Central, porción Salamanca-Irapuato, Estado de Guanajuato, México: Hermosillo, Son., Centro de Estudios Superiores del Estado de Sonora, Tesis de licenciatura, $86 \mathrm{p}$.

Orozco-Esquivel, M. T., Nieto-Samaniego, A. F. Alaniz-Alvarez, S. A, 2002, Origin of rhyolitic lavas in the Mesa Central, Mexico, by crustal melting related to extension: Journal of Volcanology and Geothermal Research, 118, 37-56.

Ortega-Gutiérrez, F., 1980, Rocas volcánicas del Maastrichtiano en el área de San Juan Tetelcingo, Estado de Guerrero. en Libro-Guía, Excursión Geol. III: Reunión Nacional Geotecnia-Geotermia, México, 34-38.

Ortega-Gutiérrez, F., 1981, Metamorphic belts of southern Mexico and their tectonic significance: Geofísica Internacional, 20, 177-202.

Oviedo de León, A., 1970. El Conglomerado Texcoco y el posible origen de la Cuenca de México: Revista del Instituto Mexicano del Petróleo, 2, 5-20.

Pasquarè, G., Ferrari, L., Garduño, V. H., Tibaldi, A., Vezzoli, L., 1991, Geologic map of the central sector of the Mexican Volcanic belt, States of Guanajuato and Michoacán: Geological Society of America Map and Chart series MCH072, 1 mapa con texto explicativo, $20 \mathrm{p}$.

Pérez-Cruz, G. A., 1988, Estudio sismológico de reflexión del Subsuelo de la Ciudad de México. México, D.F., Universidad Nacional Autónoma de México, Facultad de Ingeniería, Tesis de maestría, $83 \mathrm{p}$.

Pérez-Venzor, J. A., Aranda-Gómez, J. J., McDowell, F., Solorio-Munguía, J. G., 1996. Geología del volcán Palo Huérfano, Guanajuato, México: Revista Mexicana de Ciencias Geológicas, 13, 174-183.

Pindell, J.L., 1985, Alleghenian reconstruction and subsequent evolution of the Gulf of Mexico, Bahamas, and protocaribbean: Tectonics, 4, 1-39.

Quintero-Legorreta, O., 1992, Geología de la región de Comanja, estados de Guanajuato y Jalisco: Universidad Nacional Autónoma de México, Instituto de Geología, Revista, 10, 6-25.

Raisz, E., 1959, Landforms of Mexico: Office of Naval Research, geography branch, 1 mapa.

Riller, U., Ratschbacher, L. Frisch, W., 1992, The Tierra Colorada deformation zone: Left-lateral transtension along the northern margin of the Xolapa complex, Southern Mexico: Journal of South American 
Earth Sciences, 5, 237-249.

Rosas-Elguera, J., Ferrari, L.; Garduño-Monroy, V. H.; UrrutiaFucugauchi, J., 1996, Continental boundaries of the Jalisco Block and their influence in the Pliocene-Quaternary kinematics of western Mexico: Geology, 24, 921-924.

Ross, M. I., Scotese, C. R., 1988, A hierarchical tectonic model of the Gulf of Mexico and Caribbean region: Tectonophysics, 155, 139-168.

Salinas-Prieto, J. C., Monod, O., Faure, M., 2000, Ductile deformations of opposite vergence in the eastern part of the Guerrero Terrane (SW Mexico): Journal of South American Earth Sciences, 13, 389-402.

Schaaf, P., Morán-Zenteno, D. J., Henández-Bernal, M. S., Solís-Pichardo, G., Tolson, G., Köhler, H., 1995, Paleogene continental margin truncation in southwestern Mexico: Geochronological evidence: Tectonics, 14, 1339-1350.

Secor, D. T., Pollard, D. D., 1975, On the stability of open hydraulic fractures in the Earth's crust: Geophysical Research Letters, 2, 510-513.

Siebe, C., Rodriguez-Lara, V., Schaaf, P., Abrams, M., 2004, Geochemistry, Sr-Nd isotope composition, and tectonic setting of Holocene Pelado, Guespalapa and Chichinautzin scoria cones, south of Mexico City: Journal of Volcanology and Geothermal Research, 130, 197-226.

Silva-Romo, G., Mendoza-Rosales, C. C., Nieto-Samaniego, A. F., AlanizAlvarez, S. A., 2002, La paleocuenca de Aztlán, antecesora de la Cuenca de México, Geos, 22, 149-150.

Suter, M., Quintero, O., Johnson, C. A., 1992, Active faults and state of stress in the central part of the Trans-Mexican volcanic belt, Mexico, 1. The Venta de Bravo fault: Journal of Geophysical Research, 97, 11983-11993.

Suter, M., Quintero-Legorreta, O., López-Martínez, M., Aguirre-Díaz, G., Farrar, E., 1995, The Acambay graben: Active intraarc extension in the trans-Mexican volcanic belt, Mexico, Tectonics, 14, 1245-1262.

Suter, M., López-Martínez, M., Quintero-Legorreta, O., Carrillo-Martínez, M., 2001 Quaternary Structure of an extensional Intra-Arc-Riftthe Central Trans-Mexican Volcanic Belt: Geological Society of America Bulletin, 113, 693-703.

Takada, A., 1989, Magma transport and reservoir formation by a system of propagating cracks: Bulletin of Volcanology, 52, 118-126.

Takada, A., 1994, The influence of regional stress and magmatic input on styles of monogenetic and polygenetic volcanism: Journal of Geophysical Research, 99, 13563-13573.
Tolson, G., 1998. Deformación, exhumación y neotectónica de la margen continental de Oaxaca: datos estructurales, petrológicos y geotermobarométricos: México, D. F., Universidad Nacional Autónoma de México,Tesis doctoral, $98 \mathrm{p}$.

Tristán-González, M., 1986, Estratigrafía y tectónica del Graben de Villa de Reyes en los Estados de San Luis Potosí y Guanajuato, México: Universidad Autónoma San Luis Potosí, Instituto de Geología, Folleto Técnico, 107, $91 \mathrm{p}$.

Urrutia-Fucugauchi, J., Flores-Ruiz, J. H., 1996, Bouguer gravity anomalies and regional crustal structure in central Mexico: International Geology Review, 38, 176-194.

Urrutia-Fucugauchi, J., Ferrusquía-Villafranca, I., 2001, Paleomagnetic results for the Middle-Miocene continental Suchilquitongo Formation, Valley of Oaxaca, southeastern Mexico: Geofísica Internacional, 40, 191-205.

Vassallo, L. F., Sousa, J. E., Olalde, G., 2004, Time magmatism and mineralization at the central part of Mexico, (resumen), en 49th Annual Meeting of the Geological Association of Canada: St. Catharines, Canada, Geological Association of Canada, 385.

Venegas-Salgado, S., Herrera-Franco, J., Maciel-Flores, R., 1985, Algunas características de la Faja Volcánica Mexicana y sus recursos geotérmicos: Geofísica Internacional, 24, 47-81.

Verma, S. P., Carrasco-Núñez, G., 2003, Reappraisal of the geology and geochemistry of Volcán Zamorano, Central Mexico: implications for the discrimination of the Sierra Madre Occidental and Mexican Volcanic Belt province: International Geology Review, $45,724-752$

Watanabe, T., Koyaguchi, T., Seno, T., 1999, Tectonic stress control on ascent and emplacement of magmas: Journal of Volcanology and Geothermal Research, 91, 65-78.

Manuscrito recibido: Noviembre 01, 2004

Manuscrito corregido recibido: Marzo 17, 2005

Manuscrito aceptado: Mayo 18, 2005 